Vol. 67, N. ${ }^{\circ}$ I06 (noviembre 2015), 77-108

\title{
RACIONALIDAD DEL TRABAJO Y CAPITAL DESDE LA TASA DE GANANCIA DE MARX. UNA MIRADA (EMPÍRICA) A LAS ECONOMÍAS DE EUROPA Y NORTEAMÉRICA
}

\author{
GIOVANNY MANOSALVAS \\ Universidad Central del Ecuador
}

\author{
Recepción manuscrito: 4 de agosto de 2015 \\ Aceptación versión final: 30 de octubre de 2015
}

\begin{abstract}
RESUMEN Este trabajo constituye un acercamiento a la Ley del Descenso Tendencial de la Tasa de Ganancia propuesta por Marx a partir del análisis estadístico del Sistema de Cuentas Nacionales de los bloques económicos de la Unión Europea (15) y Estados Unidos de Norteamérica durante este último medio siglo. A partir de esto se pretende aportar algunos elementos de contrastación empírica que permitan la reflexión acerca de la racionalidad de esta dinámica en tanto se conjugan el trabajo y el capital a partir de 1) la dimensión de la disposición de los medios de producción en relación con la fuerza de trabajo, y 2) la lógica de la distribución del excedente.

palabras Clave Ley del descenso tendencial de la tasa de ganancia, Marx, plusvalía, composición orgánica de capital.
\end{abstract}

RESUMEN This work is an approach to the Law about the Tendency of the Rate of profit to Fall proposed by Karl Marx based on the statistical analysis of the National Accounts systems of the economic blocks in the European Union (15) and The United States of America during the last fifty years. Starting here, we attempt to provide some empirical contrast elements that may allow the reflection on the rationality of this dynamics while work and capital are combined in terms of 1) the dimension of availability of the means of production in relation to workforce, and 2) the logic of surplus distribution.

KEYWORDS Law about the Tendency of the Rate of profit to Fall, Marx, surplus value, organic composition of capital.

JEL CODE B51.

\section{INTRODUCCIÓN}

La noción de la caída de la tasa de ganancia no constituye en sí una tarea demasiado extenuante en el ámbito empírico, lo realmente importante, a nuestro parecer, es la interpretación de su comportamiento a la baja. Si miramos hacia atrás rápidamente, Adam Smith proponía que ello era el resultado del encuentro entre competidores, David Ricardo remitía su explicación al agotamiento de la fertilidad de la tierra, consecuentemente, a la escasez de los bienes 
alimentarios y los salarios. La corriente neoclásica (ortodoxa) explicaría el concepto de rendimientos decrecientes del capital como resultado de su agotamiento y restricción en su disponibilidad. Keynes, por su parte, basaría su explicación en torno a las condiciones del conflicto especulativo de los mercados financieros. Schumpeter afirmaría que los aparatos burocráticos de las sociedades modernas obstaculizan el trabajo de la innovación, requisito necesario para evitar su descenso. En concreto, todas estas argumentaciones concurren a espacios donde la tasa de ganancia se ve enfrentada a problemas externos a la hora de valorizar el capital, sean estos: desproporción de competencia, escases de bienes de consumo, reducción de fondos del lado de la oferta, financiarización de la economía, desaliento para la innovación, y así una larga colección de etcéteras exógenos.

Sin embargo, a diferencia de lo apuntado, Marx (contrariamente) proponía que las razones que sustentan la caída tendencial de la ganancia residirían en la propia dinámica (interna) del sistema capitalista, postulándola como una ley de carácter general. Aquí, entonces, a nuestro parecer, se sitúa el carácter rupturista de la propuesta teórica de la tasa de ganancia. Así, la discusión alrededor de la Ley del Descenso Tendencial de la Tasa de Ganancia concreta uno de los tópicos más controvertidos del legado marxista. Siguiendo a Katz (2000), el propio Marx la definió como la ley más importante de la economía política. Su proposición fue el blanco de críticas e impugnaciones desde el momento en que fue planteada en la medida en que dicha ley pone de manifiesto la caracterización de los límites históricos del capitalismo por cuando su propia dinámica interna propulsa su (auto)decadencia.

Generalmente el análisis de la tasa de ganancia se dilucida en la esfera de su comportamiento cíclico, no obstante, se suele descuidar el hecho de que, en el largo plazo, dichos ciclos sufren un descenso tendencial. Esta segunda afirmación supondría históricamente el desgaste del proceso de acumulación capitalista. No obstante, otros autores defienden la tesis de que las tasas de ganancia y el ritmo de acumulación han experimentado, más bien, procesos de crecimiento y recuperación hasta llegar a niveles de posguerra pese a la caída de los ritmos de crecimiento de las últimas décadas (Husson, 2008).

$\mathrm{Si}$, de manera intuitiva, consideramos a la generación de un valor añadido (nuevo) como premisa fundamental del proceso productivo, el concepto de tasa de ganancia supone implícitamente la asociación entre dos magnitudes: el excedente generado (creado) frente al desembolso de la inversión que el propietario de los medios (de producción) ha realizado con este fin. En otras palabras, la tasa de ganancia (g') se representa como el coeficiente entre la masa de excedente o ganancia ( $g$ ) y el monto de la inversión (i): g'=g/i. Evidentemente, en virtud de las inclusiones (o exclusiones) tanto del numerador como del denominador, existirá una variedad muy amplia de tasas de ganancia que reflejarán los diferentes matices que puedan contener las distintas medidas.

Al amparo de la Ley del Valor de Marx ${ }^{1}$ — es decir, el trabajo abstracto como fuente de valor - la tasa de ganancia se explica a partir de una premisa fundamental: el capital logra fructificarse no en base de la totalidad de la inversión que aplica, sino solo de la parte destinada a la compra de fuerza de trabajo; es decir, de la mercancía cuya caracterización particularísima consiste en la creación de un valor que excede al de su propia remuneración. La otra parte de la inversión capitalista que concreta los medios materiales (la maquinaria, materias 
primas) simplemente, traslada su valor contenido; es decir, contribuye indirectamente en la creación de valor. No obstante esta afirmación se convierte en una sentencia que pone de manifiesto una contradicción fundamental del propósito de la producción y acumulación del sistema capitalista, pues el capital — concebido en tanto medios de inversión- se encuentra limitado históricamente no por la disponibilidad física de los factores de producción (tierra, trabajo y capital), sino, por el contrario, encuentra en las relaciones de creación de valor un obstáculo insalvable: explotación del productor (de valor), el trabajador. En este momento se hace imprescindible determinar con absoluta claridad que la construcción de todo el aparataje teórico, sustento de la Ley del Descenso Tendencial de la Tasa de Ganancia, es posible (sí y solo sí) bajo la articulación de la Ley del Valor (de Marx), caso contrario ninguna explicación derivada tendría sentido. ${ }^{2}$

Por otro lado, las tensiones de tecnificación causadas por un permanente estado de competencia configuran un escenario productivo donde la fracción del capital que se orienta a la compra de fuerza de trabajo tiende a perder su peso relativo frente a su contraparte - la fracción del capital sufragada en medios de producción o capital constante-como resultado de un proceso sostenido de mecanización del trabajo. Es así como a través de la lógica capitalista subyace una tendencia al incremento de la productividad del trabajo a la par de una disminución de la participación de los salarios en el proceso productivo, fruto del irrefutable avance tecnológico. Así, este efecto dinámico (contradictorio) configura la caída tendencial de la tasa de ganancia en virtud de que quien crea valor va siendo, sistemáticamente, sustituido por quien no lo crea, sino lo transmite. ${ }^{3}$ No obstante, atendiendo a estas contradicciones, la estabilidad o, inclusive, el crecimiento de la tasa de ganancia supone la incursión de dinámicas y/o factores contrarrestantes que, en términos estructurales, no anulan este posible descenso tendencial.

El análisis de la Ley del Descenso Tendencial de la Tasa de Ganancia, a la par del debate teórico de partida, ha concertado contemporáneamente importantes investigaciones ${ }^{4}$ sobretodo en torno a su posible aproximación empírica. Un referente ineludible es, sin duda, Measuring the Wealth of Nations: The Political Economy of National Accounts, de A. Shaikh y A. Tonak (1994). Estos esfuerzos han culminado en una serie de propuestas metodológicas para el análisis de las categorías marxistas a través del estudio del Sistema de Contabilidad Nacional (estadísticas y/o cuentas nacionales).

En este marco, nuestro trabajo pretende poner de manifiesto la validez de la dinámica del descenso tendencial de la tasa de ganancia y su potencia argumentativa para caracterizar la lógica del sistema de producción capitalista y sus relaciones causales desde el ámbito empírico. El periodo de estudio abarca la evolución de las economías de los países de la Unión Europea (15) y Estados Unidos de Norteamérica entre 1960 y 2010. Se considera, pues, que la pertinencia de nuestra contribución, al final, se traduce en aportar a la discusión elementos de contrastación empírica a uno de los apartados teóricos más importantes del aporte marxista: La Ley del Descenso Tendencial de la Tasa de Ganancia. Para este fin dicha tasa cualitativamente se desmantela en: i) la composición orgánica del capital; 5 es decir, una dimensión de la disposición de los medios de producción en relación con la fuerza de trabajo; y ii) la tasa de plusvalía; es decir, la dinámica de la distribución del excedente; en otras palabras, la relación entre la plusvalía (ganancia) y el capital variable (fuerza de trabajo). De este modo, mediante la adopción 
de varios ajustes metodológicos, se intenta recrear empíricamente la propuesta teórica de Marx a través de la revisión del Sistema Nacional de Cuentas.

En concreto, este trabajo empírico procura dotar de ciertos medios (elementos analíticos) que posibiliten al final dar respuestas a: i) ¿es plausible corroborar una caída tendencial de la tasa de ganancia? Si es así ¿cuáles son las causas que explican ese descenso?, caso contrario ¿qué argumentos soportan su estabilidad e, inclusive, su ascenso?; y ii) atendiendo a sus elementos constitutivos, ¿ es posible determinar una tendencia a la mecanización del proceso productivo que se exprese finalmente en un aumento del peso del capital constante respecto al variable? De ser así, ¿cuáles son los efectos derivados en la productividad del trabajo y apropiación del excedente? y ¿cuáles son los impactos en términos de tasa ganancia? Se pone, entonces, a prueba la hipótesis de que, en base al análisis empírico de las economías de la Unión Europea (15) y Estados Unidos de Norteamérica, se verifican una serie de elementos compatibles con la formulación teórica de la Ley del Descenso Tendencial de la Tasa de Ganancia.

\section{EL CONCEPTO DE TASA DE GANANCIA EN MARX ${ }^{6}$}

Para entender algunas de las premisas más importantes que sirven de soporte al análisis marxista es necesario conceptualizar el proceso productivo desde su composición más básica. Así, tenemos que el esquema de fructificación capitalista queda genéricamente representado por D-M...P...M'-P', donde una cantidad inicial de dinero (D) adquiere mercancías (M) cualitativamente distintas que pasan a materializar medios de producción y fuerza de trabajo y que se combinan dentro del proceso productivo (P) dando como resultado una mercancía distinta (M') poseedora de un valor nuevo que, en su fase de realización, se convertirá en un valor dinerario (D') superior al inicial (D). El paso de м a M'; es decir, la creación de valor nuevo (plusvalía, pv), materializa la fuente de la ganancia, de este modo Marx alude al fundamento de la acumulación capitalista: la búsqueda incesante de la ganancia en relación con el capital invertido (adelantado); es decir, la tasa de ganancia (g') o (D'-D)/D.

Como complemento de lo anterior, cabe detenernos en una diferenciación esencial que pasa por el carácter cualitativo de las mercancías que entran en el proceso productivo: medios de producción (MP) y fuerza de trabajo (FT). A la luz de la Ley del Valor de Marx, solamente la mercancía que tiene la capacidad de incrementar el valor del capital adelantado es la fuerza de trabajo (FT); es decir, el trabajo vivo que constituye el trabajo presente, líquido, no materializado en ningún proceso productivo. Consecuentemente todos los restantes componentes plasmados en los medio de producción (MP) carecen de esta posibilidad aumentadora del valor convirtiéndose en únicamente trasmisores de su valor contenido. Por lo tanto, la obtención de la plusvalía se dilucida en la mercantilización de la fuerza de trabajo conjuntamente con la colaboración indirecta de los medios de producción.

La fracción del capital adelantado que se destina a la adquisición de los medios de producción únicamente transmite su mismo valor a las nuevas mercancías (no contempla creación de valor alguno); sin embargo, la fracción del capital que se invierte en fuerza de trabajo reproduce su mismo valor añadiendo un nuevo margen. La diferencia entre el capital adelantado que se destina al pago de la fuerza de trabajo y lo que ésta realmente produce se conoce 
con el nombre de plusvalía (pv) o, dicho de otro modo, la diferencia entre el trabajo producido y el trabajo sufragado por el capitalista es el trabajo no pagado que sintetiza la esencia de la explotación, elemento consustancial del sistema de producción capitalista. En apego a las posibilidades cualitativas del capital adelantado en medios de producción (MP) y fuerza de trabajo (FT) se denomina, respectivamente, capital constante (c) y capital variable (v). La relación entre estas dos expresiones, dentro del proceso productivo, se conoce bajo el nombre de composición orgánica del capital o c/v, aludiendo a las condiciones técnicas bajo las cuales se combinan los medios de producción y el trabajo.

Hasta aquí hemos presenciado el ciclo de producción capitalista de manera esquemática bajo una perspectiva estática, no obstante como lo afirma Gill (1996) «la observación de la instantánea (fotográfica) de la producción del valor debe completarse con la visión de la película de su reproducción ininterrumpida» (p. 312); es decir, cabría entender al funcionamiento del sistema capitalista al amparo de su representación dinámica de la realidad que visibilice el movimiento contenido en sus fuerzas.

Esta concepción holística del proceso productivo que involucra a la creación permanente de un nuevo excedente (plusvalía) capaz de regenerarse, asume intrínsecamente el hecho de que el circuito D-M...P...M'-D' tiene que ser renovado y además, forzosamente, ampliado. Así, después de la reposición de los medios consumidos, ${ }^{7}$ una parte del excedente (plusvalía) se encuentra obligada a alimentar al siguiente proceso productivo configurándose su crecimiento o reproducción ampliada. Esta afirmación se sostiene bajo el hecho de que el capital, en cualquiera de sus expresiones (en este momento dineraria), no tiene otra finalidad que ser fructificado por la acción del capitalista. Aparentemente el destino del excedente obedece a la voluntad de los capitalistas (inversores); sin embargo, la presión de la competencia juega un papel decisivo que, al final, marca los patrones conductuales de las: i) reinversiones (nueva acumulación); y ii) consumo improductivo. Concretamente, el circuito D1-M1...P1...M1'-D1' se convertirá en D2-M2...P2...M2'-D2', manteniendo su carácter general, cuando D2>D1.

Una vez conocidos estos antecedentes estamos en condiciones de abordar el análisis de la tasa de ganancia (eje teórico vertebrador de nuestra investigación). La tasa de ganancia, concebida genéricamente en términos de la relación entre un capital cualquiera y su correspondiente fructificación o rendimiento es, sin duda, el móvil de la acumulación, se constituye en sí misma su fuerza motriz, es, dicho de otro modo, el estimulante de la producción. Por esta razón, el índice de movilidad de los capitales para desplazarse intra o intersectorialmente de los espacios con bajas tasas de beneficio a aquellos que ofrecen beneficios mayores configura una condición de estabilidad sistémica. Así, una rama o sector que tiene una producción sobreabundante tendrá, consecuentemente, problemas de realización que, pensando sobre los precios de mercado, originará localmente una disminución de la tasa de ganancia efectivamente realizada. En consecuencia, los capitales se verán motivados a orientarse hacia otras ramas o sectores teniendo por efecto una reproducción reducida del capital y, finalmente, una disminución de la producción en la rama inicialmente excedentaria. Si esta reducción es, por ejemplo, excesiva, los precios de los bienes o los servicios, bajo la lógica de la oferta y demanda, tienden a aumentar y con ellos su tasa de ganancia, lo que estimulará (nuevamente) la reubicación de los capitales en procura de su mejor fructificación. 
No obstante, volviendo a la noción clásica de la tasa de ganancia de Marx, el valor final total del proceso productivo es $\mathrm{V}=\mathrm{c}+\mathrm{v}+\mathrm{pv}$, donde el valor total $(\mathrm{V})$ que corresponde a la suma de los capitales constante (c) y variable (v) más la plusvalía (pv). Por lo tanto la tasa de ganancia (g') puede expresarse en términos de $\mathrm{pv} /(\mathrm{c}+\mathrm{v})$. Cabe mencionar que, conforme con la Ley del Valor de Marx, la única fracción de capital creadora de valor nuevo es la fuerza de trabajo o capital variable (v), por lo tanto la evolución de la tasa de ganancia pasa a depender de la proporción relativa del capital constante o medios de producción (c) y el capital variable o la fuerza de trabajo (v); es decir, de la composición orgánica del capital (c/v).

Otra consideración importante se desprende del hecho de que cuanto mayor sea la proporción del capital variable (única fuente de creación) que genera efectivamente valor nuevo respecto de su costo, mayor será la extracción de plusvalía en relación con todo el capital. Dicho de otra forma, cuanto más grande sea la brecha entre el trabajo producido y el trabajo realmente pagado, en relación con el capital invertido, mayor será la tasa de ganancia. La proporción, cuota o tasa de plusvalía (pv'), por tanto, se manifiesta a través de la relación entre la masa de plusvalía (pv) y el elemento que lo genera; es decir, el capital variable (v) siendo pv'=pv/v. Así la relación entre la plusvalía y la ganancia es positiva. Así tenemo la Tasa de plusvalía-tasa de ganancia (Gill, 2002, p. 213):

$$
g^{\prime}=\frac{p v}{c+v} \Rightarrow g^{\prime}=\frac{p v^{\prime}}{\left(1+\frac{c}{v}\right)} \Rightarrow\left\{\begin{array}{l}
\frac{\partial g^{\prime}}{\partial p v^{\prime}}>0 \\
\frac{\partial g^{\prime}}{\partial c / v}<0
\end{array}\right.
$$

Así, la tasa de ganancia (g’) mantiene una relación positiva con la tasa de plusvalía (pv') e inversa con la composición orgánica del capital (c/v). La noción de tasa de plusvalía pv/v (o dicho a la inversa, el salario relativo, v/pv), pone de relieve que lo más importante en el capitalismo no es la evolución del salario nominal —el precio de la fuerza de trabajo en moneda nacional - ni la del salario real —el precio nominal de la fuerza de trabajo en relación con el precio de las mercancías que consumen los trabajadores-, sino el peso relativo de la plusvalía y salarios cuya inversa es el salario relativo - el ratio que pone en relación el salario con la ganancia o plusvalía-, (Arrizabalo, 2014, p. 326).

Siguiendo lo anterior, cabe destacarse que el salario real podría experimentar, sin problema, ciertos momentos de crecimiento; sin embargo, un incremento del salario relativo supondría poner el riesgo la misma continuidad del proceso de acumulación y reproducción del capitalismo, por cuanto ello constituye una imposibilidad sistémica. Esta concepción sobre las limitaciones del salario constituye en sí mismo uno de los elementos centrales del planteamiento marxista.

\section{LEY DEL DESCENSO TENDENCIAL DELA TASA DE GANANCIA Y SUS CAUSAS}

El proceso dinámico de producción (re-producción) capitalista se soporta sobre el hecho de que una parte de la plusvalía obtenida, obligatoriamente, tiene que materializarse en nuevo capital 
(capitalización de la plusvalía), lo que supone una nueva asignación eficiente para adquirir medios de producción (capital constante) y fuerza de trabajo (capital variable). Esta tendencia de movimiento continuo configura dos efectos: i) inestabilidad en las proporciones del capital constante y variable; y ii) posiblemente la más importante, la sustitución continua de la fuerza de trabajo por medios de producción. ${ }^{8}$ Esta última aseveración, además, ratificada por Katz (2002).

Marx sostiene que, en el contexto del sistema de producción capitalista, existe una marcada tendencia a la acumulación de los capitales, así como también, paralelamente, subsiste la propensión al alza de la composición del capital. Por lo tanto, es claro que si c/v tiende a incrementarse, la relación pv/(1+(c/v)) decrecerá.

La sustitución de fuerza de trabajo por medios de producción fundamenta la continua mecanización del proceso de producción a efectos de mejorar los niveles de productividad con el objetivo de maximizar el beneficio y así fructificar al capital. Este principio marca una de las pautas consustanciales para el sistema de producción capitalista: su mecanización creciente. No obstante, el hecho de que el propio sistema capitalista disminuya, en términos relativos, a la única fuente de creación de plusvalía, el trabajo humano, constituye una de sus mayores contradicciones, ya que la creación de plusvalía no es fruto del consumo de los dos tipos de mercancías (fuerza de trabajo y medios de producción), sino solamente de la acción directa del trabajo vivo.

En palabras más simples. Si suponemos, por ejemplo, que la tasa de plusvalía (pv’) permanece constante, la tasa de ganancia ( $\mathrm{g}$ ) varía en sentido inverso a la composición orgánica del capital (c/v); es decir, si ésta tiende a crecer, la tasa de ganancia caerá. Sin embargo, conocemos que la composición orgánica del capital tiende a crecer en el curso del desarrollo capitalista, por lo tanto, debe existir al menos una tendencia compensadora para evitar que la tasa de ganancia (g') descienda efectivamente. Esta premisa constituye uno de los aportes más importantes del cuerpo teórico de Marx: la tendencia secular del descenso de la tasa de ganancia.

Con la progresiva disminución relativa del capital variable con respecto al capital constante, la producción capitalista genera una composición orgánica crecientemente del capital global, cuya consecuencia directa es que la tasa del plusvalor, manteniéndose constante el grado de explotación del trabajo e inclusive si éste aumenta, se expresa en una tasa de ganancia constantemente decreciente [...]. (Marx, 1894, p. 271)

Según la tradición marxista clásica, la Ley del Descenso Tendencial de la Tasa de Ganancia subsiste sin cesar; sin embargo, no se materializa forzosamente en la realidad por un descenso efectivo, esto como resultado de ciertos efectos que pueden contrarrestar esta tendencia. ${ }^{9}$ Bajo este argumento se afirma que esta tendencia expresa un conjunto de relaciones entre la acumulación de capital, la composición orgánica del capital, los progresos en la productividad del trabajo y el grado de explotación de la fuerza de trabajo.

CAUSAS CONTRARRESTANTES DE LA LEY DEL

DESCENSO TENDENCIAL DE LA TASA DE GANANCIA

A efectos de evitar la caída efectiva de la tasa de ganancia, como derivación del aumento de la composición orgánica de capital que, dicho sea de paso, materializa el progreso de la 
productividad social, existen ciertas influencias que permiten que esta ley se manifieste de manera tendencial; es decir, que sea plausible considerar que su descenso se mantenga contenido debido a circunstancias contrarrestantes. En este sentido, Katz (2002) afirma:

Marx puntualizó al mismo tiempo la existencia de seis fuerzas compensatorias de este proceso. El aumento del grado de explotación (plusvalía absoluta obtenida a través de la intensificación o prolongación de la jornada de trabajo), la reducción del salario por debajo de su valor, el abaratamiento del capital constante (derivado de la depreciación del capital existente), la superpoblación relativa (abundancia del trabajo asalariado disponible para incrementar la explotación o reducir el salario), el comercio exterior (favorable a los países desarrollados mediante el abaratamiento de las materias primas y los bienes de consumo de los asalariados) y el aumento del capital por acciones (cuyos dividendos permiten ganancias extraordinarias). Marx destacó, además, que el incremento de la capacidad productiva, del volumen de las ventas y de la masa total de ganancias contrarrestan, pero no revierten la caída de la tasa de beneficio. (p. 6)

De esta manera, ante una caída tendencial de la tasa de ganancia los capitalistas se mantendrán obligados a reaccionar en varios sentidos, por ejemplo, un aumento de sus esfuerzos con el fin de restablecer la rentabilidad de sus negocios a través de elevar la productividad laboral - reorganización de la tareas, incremento de los ritmos, acrecentamiento de las labores de inspección, disminución de los tiempos de descanso, relajamiento de las normas de seguridad e higiene-, así como también la exigencia de un marco legislativo favorable a los empleadores/ capitalistas — congelamientos salariales, reducción de la reglamentación laboral, eliminación del derecho a huelga, caída del salario mínimo-, etc. Además, es posible diferenciar las contratendencias que apuntan en los dos sentidos: la extracción de plusvalía (explotación y sobrepoblación) de los que procuran una baja composición orgánica del capital (su abaratamiento) o, un tercer escenario alternativo, que contendría a la convergencia de estos dos vectores (la palanca del comercio exterior).$^{10}$

A nuestro entender, la materialización de estas fuerzas contrarrestantes termina concretándose, en última instancia, mediante: una desvalorización del capital constante, el uso del comercio exterior, el aumento del capital por acción y/o, fundamentalmente, la presión sobre el grado de explotación (pv') que relaciona el valor creado con su fuerza creadora (en valor) de pago. Así, el aumento del nivel de explotación se sujetaría, por tanto, a dos vertientes: i) a través del incremento de la plusvalía absoluta que consiste en la reducción de salario por unidad de tiempo que bien podría establecerse al aumentar la jornada laboral o al disminuir el salario; es decir, propender a un aumento relativo de plustrabajo; ${ }^{11} \mathrm{y}$ ii) mediante la ampliación de la plusvalía relativa que radica en el aumento en la productividad de los trabajadores que, evidentemente, disminuye el tiempo de trabajo utilizado para la retribución de los salarios aumentando, igual que en el caso anterior, el peso relativo del plustrabajo en la jornada laboral. ${ }^{12}$

En esta perspectiva, recordando la formulación genérica de la tasa de ganancia $g^{\prime}=\mathrm{pv}^{\prime}(1+(\mathrm{c} / \mathrm{v}))$ podemos situar la discusión sobre un requisito indispensable para, al menos, impedir el descenso de la tasa de ganancia: el aumento de la tasa de plusvalía debe contrarrestar (o ser mayor) al incremento de la composición orgánica del capital. Formalizando esto tenemos: 


$$
\Delta g^{\prime} \geq 0 \rightarrow \frac{\partial g^{\prime}}{\partial p v^{\prime}} \geq \frac{\partial g^{\prime}}{\partial \frac{c}{v}}
$$

Resumiendo esta contradicción de manera más simple, la problemática de la caída tendencial de la tasa de ganancia radica sobre el hecho de que cada vez existen más presiones sobre la obtención de plusvalía (pv) a la par de que se minimiza el trabajo que la genera (v).

La tasa de ganancia no disminuye porque el trabajo se haga más improductivo, sino porque se torna más productivo. Ambas cosas, tanto el aumento en la tasa del plusvalor como la baja en la tasa de ganancia, sólo son formas particulares mediante las cuales se expresa en el modo capitalista de producción la creciente productividad del trabajo. (Marx, 1867, p. 307)

Hasta aquí, de manera breve, hemos tratado de sintetizar algunos de los aportes más relevantes de la Ley del Descenso Tendencial de la Tasa de Ganancia —en su estado más puro- de cara al análisis aplicado/estadístico. Consideramos, además, que es menester aclarar que la discusión teórica tiene un recorrido muy rico en tanto a sus debates, elementos explicativos y relaciones causales; ${ }^{13}$ no obstante, este primer acercamiento conceptual, sin que de modo alguno se encuentre zanjado, ${ }^{14}$ permite ubicar algunas herramientas analíticas que constituyen insumos indispensables para hilar el trabajo aplicado.

\section{TRABAJO APLICADO: MARCO METODOLÓGICO Y CONSTRUCCIÓN DE EQUIVALENCIAS}

En primer lugar, es imperativo explicitar los aspectos que, en estricto apego a los objetivos de nuestro trabajo de investigación, se excluyen: i) la reacomodación del cuerpo teórico de Marx en vista de la multiplicidad de debates académicos surgidos a raíz del problema de la extrapolación de los datos de las cuentas nacionales hacia las categorías marxistas; ii) una recomposición de las cuentas nacionales en estricto apego a los fundamentos de la teoría marxista; y iii) una explicación exhaustiva sobre la evolución de los bloques económicos examinados. La explicación de los aspectos (excluidos) i y ii que, evidentemente no son menos importantes, responde a que la intención primaria del trabajo aplicado es, a partir de los supuestos neoclásicos que sirven de base para la construcción de las cuentas nacionales, encontrar elementos de contrastación empírica compatibles con la propuesta de la Ley del Descenso Tendencial de la Tasa de Ganancia de por Marx. Ello, a primera vista, no está exento de controversias; sin embargo, consideramos que a través del uso de las herramientas disponibles en las cuentas nacionales y mantenidas, por así decirlo, en su estado más puro — es decir, sin mayores arreglos o contaminaciones - , existe el potencial analítico para generar algunas explicaciones o aproximaciones sobre la dinámica capitalista. En lo relativo a la exclusión iii, debemos afirmar que si bien la acotación del trabajo aplicado exige remitirnos de manera tangencial a algunas referencias históricas muy generales debido a la heterogénea composición de los bloques - especialmente en alusión al bloque de la Unión Europea (15)—; en rigor, una explicación (más) 
específica desbordaría el carácter generalizante de las dinámicas explicativas de ambas economías que, intencionalmente, se pretende abordar.

En segunda instancia, la variable de estudio constituye, pues, la tasa de ganancia y, por tanto, eje transversal de nuestro análisis. Este baremo, en tanto indicador, acorde al planteamiento teórico marxista, como se ha afirmado, determina las pautas del proceso de acumulación. Así, entonces para abordarlo se plantea la construcción de varios indicadores para las economías de los países de la Unión Europea (15) y de los Estados Unidos de Norteamérica durante el periodo de tiempo 1960-2010. Éstos, construidos a partir de la contabilidad nacional, pretenden reflejar aproximaciones a las categorías marxistas, realizadas mediante la adopción de varios arreglos metodológicos.

Siguiendo la propuesta de Shaik (1984), instrumentalmente el concepto de tasa de ganancia (en general) y los demás elementos analíticos (en particular) pueden ser estudiados (observados) a través de sus equivalentes relativos, siendo: la masa del excedente bruto de explotación (EBE) como un indicador de la masa de plusvalía (pv), la masa de remuneraciones de los asalariados (RA) como una aproximación del capital variable (v), el stock neto de capital (K) como un indicador congruentemente compatible con la categoría de acervo de capital (c) y, además, el número de trabajadores (L) que, evidentemente, no contempla arreglo alguno. A partir de ello se calculan los índices: la tasa de beneficio ${ }^{15}(\mathrm{EBE} / \mathrm{K})$ como un proxy de la tasa de ganancia $\left(\mathrm{g}^{\prime}\right),(\mathrm{K} / \mathrm{L})$ como un proxy de nivel de capitalización de la economía, ${ }^{16}$ (L/PIB) como un acercamiento a la productividad del trabajo, (EBE/PIB) como una representación de la pugna distributiva o su opuesto (RA/PIB) que encarna el concepto de salario relativo, y (EBE/RA) como una medición cercana de la tasa de plusvalía (pv'). Con base en el análisis de la evolución de estos indicadores se concretan varias reflexiones intentando aportar elementos de discusión de cara a los postulados propuestos por Marx en la Ley del Descenso Tendencial de la Tasa de Ganancia.

A la luz de esta propuesta de tratamiento empírico, surgen inmediatamente varias interrogantes: ¿Es posible, a través de la contabilidad nacional, considerando las limitaciones metódicas, metodológicas y de fundamentación, abordar un análisis riguroso de las categorías propuestas por Marx? Es decir, a través de las cuentas nacionales, ¿es factible visibilizar los aportes teóricos marxistas contenidos en la Ley del Descenso Tendencial de la Tasa de Ganancia? o, atendiendo estas incompatibilidades, ¿es susceptible la adopción de instrumentos metodológicos que aproximen la información contenida en las estadísticas nacionales a los postulados marxistas? En efecto. No obstante, la admisión de estos mecanismos de translocación supone inconvenientes no menores; aunque consideramos de obligatorio cumplimiento sobre todo si se trata de encarar un análisis empírico basado en las estadísticas convencionales.

Ciertamente, la más importante incompatibilidad, y por tanto todas sus derivaciones metodológicas, se concreta en torno al presupuesto teórico (analítico) de partida que sustenta la base de toda la información contenida en las cuentas nacionales ${ }^{17}$ que, claramente, no es compatible con la formulación marxista por cuanto desde el universo neoclásico, desde donde se articula la contabilidad nacional, se advierte que los factores productivos - por citar: tierra, capital y trabajo - reciben el equivalente justo de sus aportes dentro del proceso productivo; esta fundamentación keynesiana deja de lado la noción de explotación propuesta por Marx que, por citarlo brevemente, rebela la existencia de un valor no pagado (expropiado) al trabajador 
que, por tanto, es apropiado por el capitalista. ${ }^{18}$ Por consiguiente, la contabilidad nacional no realiza (ni necesita tampoco) distinciones entre: trabajo productivo e improductivo, valor capitalista y no capitalista, actividades mercantiles y no mercantiles, que constituyen, pues, insumos primordiales del análisis marxista. No obstante, existen varios arreglos metodológicos que razonablemente permiten una cierta aproximación de las estadísticas nacionales a las categorías estudiadas por Marx. Este proceso de transición exige asumir, indefectiblemente, ciertos desfases gestionados de manera controlada.

En atención a lo que sostiene Shaikh (2011) respecto de las limitaciones del uso de fuentes o estadísticas convencionales, ${ }^{19}$ estamos de acuerdo en que si bien existe una diferencia sistemática entre la estructura teórica de análisis neoclásico y la del análisis marxista (más ortodoxo), es comprensible que en la trayectoria de lo abstracto hacia lo concreto esta diferencia sea (relativamente) infranqueable; sin duda, los principios, métodos y categorías son diferentes, el propósito en sí mismo es distinto, por definición el resultado final será necesariamente diferente; no obstante, consideramos que en vez de despreciar esta fuente de información se podría llevar a cabo un replanteamiento del análisis a fin de convertirlo en un instrumento capaz de rebelar algunas relaciones del sistema capitalista de producción. De esta manera se vislumbra, en principio, una posibilidad real a la hora de utilizar la información (intacta) de las cuentas nacionales para el análisis marxista.

Otro argumento que apuntala el uso de las cuentas nacionales y sus relativas consonancias con el análisis marxista, se fundamenta en base a la descripción del ciclo del capital productivo realizado por Shaikh (1984), que propone, en un primer nivel de abstracción —es decir, como punto de partida-, la posibilidad de relacionar al capital variable (vM) con la masa salarial (RACN) y a la plusvalía (pvM) con las rentas del capital (EBECN). Ello supone una relación de equivalencia de la tasa de plusvalía $\mathrm{pv}^{\prime} \mathrm{M}=(\mathrm{pv} / \mathrm{v})$ con la razón ganancia salario ${ }^{20} \phi \mathrm{CN}=(\mathrm{EBE} /$ $\mathrm{RA})$. De este modo, puede establecerse que pv' $\approx \phi \mathrm{CN}$. Donde los subíndices $\mathrm{M}$ representan las tesis marxistas y los subíndices $\mathrm{CN}$ las medidas de las cuentas nacionales - la revisión detallada de estos conceptos y el marco general se verán más adelante-. Es importante mencionar, además, que los subsecuentes análisis llevados adelante por Shaikh (1984), a partir de esta primera consideración, concluyen en un proceso de disgregación extremadamente minucioso lo que permite (al final) que las cuentas nacionales se aproximen con mayor correspondencia a las categorías marxistas. ${ }^{21}$ No obstante, la intencionalidad particular de este trabajo de investigación empírica, plantea el uso de la contabilidad nacional en su estado más puro para, a partir de ello, llegar a inferir algunas compatibilidades con Ley del Descenso Tendencial de la Tasa de Ganancia. La utilización de las cuentas nacionales como elementos de referencia, dentro del análisis marxista, ha concertado la atención de varios investigadores cuyos trabajos han culminado en tesis doctorales; véase Guerrero (1989), Cámara (2003) y Mateo (2007). Del mismo modo, podemos encontrar publicaciones que contienen aportaciones y debates en el sentido teórico y del tratamiento empírico. Algunos referentes son: Shaikh, A. (1984), Mandel, E. (1985), Katz C. (2000), Tomé, J. P. M. (2007), Cámara I. y Mariña F. (2010), Heller, P. (2011), Maito, E. E. (2014), entre otros.

Por otro lado, a fin de desplegar el trabajo empírico se ha considerado apropiado utilizar la fuente estadística $\mathrm{AMECO}^{22}$ en virtud de varias ventajas: i) contiene las bases de datos 
macroeconómicos, con una periodicidad anual, gestionada por la Dirección General de la Comisión Europea para Asuntos Económicos y Financieros (DG ECFIN); ii) ofrece series temporales desde 1960; y iii) recoge la información de los bloques económicos norteamericano y europeo (15) lo que constituye un marco de comparación y, posteriormente, de generalización. Concretamente, y en función de las consideraciones anteriores, se plantea asumir los siguientes supuestos: i) la masa del excedente bruto de explotación (EBE) puede mantener mayoritariamente similitudes con la masa de las rentas del capital; es decir, con la plusvalía (pv); ii) la masa de remuneraciones de los asalariados (RA) puede reflejar similitudes importantes con el capital variable (v); y iii) el stock neto de capital puede ser congruentemente compatible con la categoría de acervo de capital (c). Estas tres consideraciones metodológicas (de partida) se plantean con la pretensión de utilizar la contabilidad nacional pura con el fin (pretendido) de compatilibilizarla con la Ley del Descenso Tendencial de la Tasa de Ganancia para así advertir el carácter de su vigencia.

Por último, es necesario precisar categóricamente que a través de estos supuestos simplificadores, que soportan al consecuente análisis empírico, se asumen ciertas premisas que son, obviamente, potencial fuente de discusión. No obstante, la adopción de estas condiciones de partida permite una aproximación razonablemente fiable a los postulados propuestos por Marx que, de otra forma, no serían posibles.

\section{TASA DE ACUMULACIÓN DE LA ECONOMÍA: INVERSIÓN VS STOCK DE CAPITAL (FNKF/K)}

Con el fin de estructurar un análisis pretendiendo un cierto grado de complejidad en cada una de las explicaciones proponemos, en primera instancia, introducir el estudio de la tasa de ganancia revisando un primer elemento determinante: el ritmo de acumulación ${ }^{23} \mathrm{O}$ velocidad de regeneración del sistema de producción desde las perspectivas de la inversión, el stock de capital y el PIB.

La capacidad de acumulación (regeneración) del sistema de producción capitalista puede aproximarse mediante el comportamiento de la tasa de acumulación que sintetiza la relación entre la formación neta de capital fijo (FNKF) con el stock de capital (K); es decir, la cantidad de recursos empresariales que se inyectan en la economía en relación con el capital acumulado (ver Figura 1).

De lo observado se desprenden dos indicios. Por un lado se visibiliza, para el caso de ambos bloques económicos, la sensibilidad de la tasas de acumulación a los ciclos económicos evidenciando la variabilidad de la aceleración del proceso productivo. En segunda instancia, se constata un descenso tendencial de las tasas de acumulación a largo plazo (líneas segmentadas) aunque con una pendiente mucho más pronunciada para el caso de la economía de la UE-15. Estas aseveraciones pueden entenderse a la luz de la evolución de las tasas promedio (decenales) de acumulación. Así en los países de la UE-15 presenciamos cinco décadas de descenso ininterrumpido durante los años 60 (4,76\%), 70 (4,34\%), 80 (2,42\%), 90 (2,26\%) y dos mil (2,04\%). De manera similar, aunque con una caída más tenue, durante tres de sus primeras cinco décadas, la economía norteamericana experimenta, un descenso tendencial de sus tasas promedio de acumulación a lo largo de los años 60 (2,77\%), 70 (2,76\%) y 80 (2,46\%). No obstante, en la década de los 90 la tasa de acumulación promedio $(2,64 \%)$ rompe su ciclo 
Figura 1. Tasas de variación interanual del PIB y de la formación neta de capital fijo (FNKF), UE-15

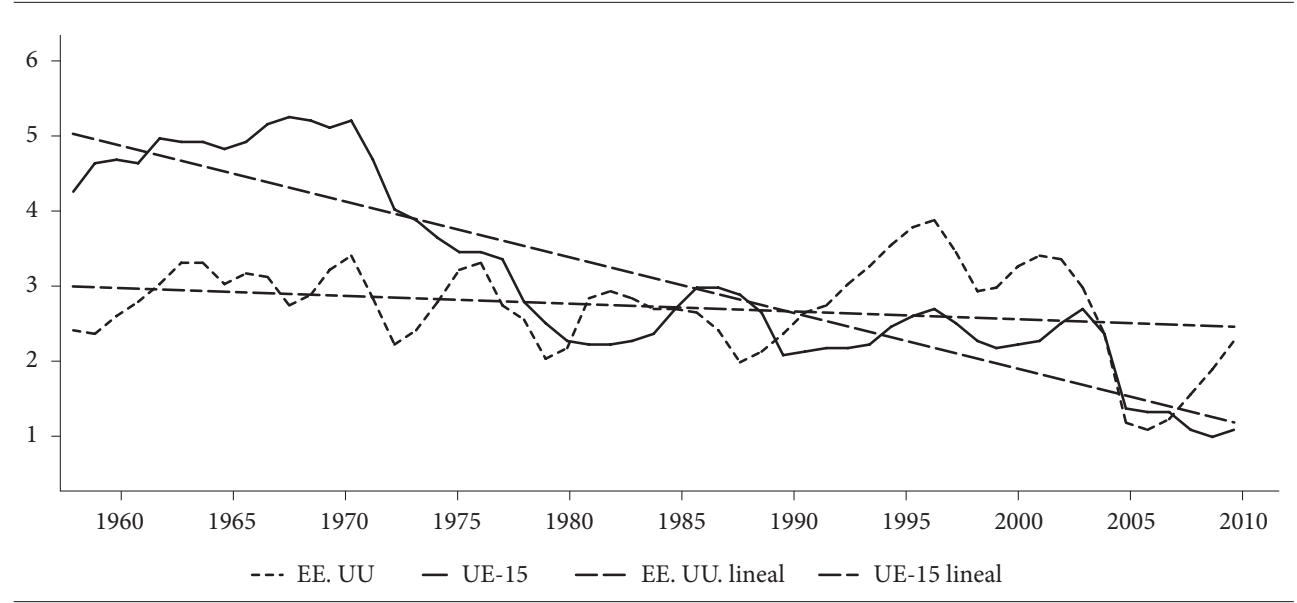

Fuente: Elaboración propia a partir de los datos de AMEco.

decreciente en relación a sus tres décadas anteriores como resultado de tasas ascendentes registradas desde 1991 hasta 1999; sin embargo, una nueva tendencia decreciente hace su aparición en la década de los dos mil exceptuando el periodo 2003-2005, que recoge un crecimiento de estas tasas; sin embargo, la década del dos mil, vista desde una panorámica decenal, supone un proceso de caída del ritmo de acumulación. Dicho esto, en principio, podemos corroborar que la economía norteamericana mantiene una caída tendencial de su tasa de acumulación en cuatro de sus cinco décadas.

Concretamente, a la luz de estos resultados, para ambos bloques, se detecta, al menos en principio, ciertas pautas que determinan una marcada incapacidad del sistema de producción capitalista para re-generar condiciones estables (aún más crecientes) de acumulación. Esta afirmación sienta un primer e importantísimo precedente.

\section{TASA DE ACUMULACIÓN DE LA ECONOMÍA: PIB VS. INVERSIÓN (FNKF) $)^{24}$}

Como complemento del análisis del apartado anterior, consideramos pertinente, además, abordar una segunda discusión respecto del ritmo de acumulación pero desde otra perspectiva que parte de la relación entre el comportamiento del PIB y la FNKF —entendida, para el efecto, como un vector de inversión empresarial-. Retomando el hecho de que la lógica del sistema de reproducción capitalista entraña que, una vez que se reponen los medios consumidos en el proceso productivo; es decir, la fuerza de trabajo y los medios de producción, el residuo resultante (excedente) tiene dos alternativas: canalizarse hacia un consumo improductivo o reinvertirse como nuevo capital y es, precisamente, en el proceso de reinversión donde se funda uno de los principios esenciales de la acumulación capitalista que concreta no solo el ánimo productivo de los capitales, sino, además, la presión competitiva entre ellos. 
La importancia del vector formación neta de capital (FNKF) radica en que, de algún modo, éste condensa el movimiento empresarial en tanto representa la masa de recursos reinvertidos efectivamente en la economía por parte de los capitales. Complementariamente, otro de los termómetros del crecimiento de la actividad económica es, cotidianamente, la evolución del PIB. Ambos indicadores proveen, también, una aproximación al proceso de reproducción y acumulación del capital (ver Figura 2).

De la observación anterior, podemos intuir, en términos de evolución del PIB, respecto de la economía de los países de la Unión Europea (15), tres premisas: i) la presencia de periodos de crisis lo que manifiesta, de alguna manera, la incapacidad del sistema de producción capitalista para mantener un crecimiento equilibrado de largo aliento, así se confirma en 1975, 1993, 2009 y 2012 (crisis derivada de 2009); ii) los procesos de recuperación de las crisis se manifiestan cada vez menos intensos - expresado en la tasa de variación interanual promedio de los tres años de recuperación-, así, el periodo de crisis de 1975 lleva aparejado un 3,42\%, el de 1993 un 2,32\%, el de 2009 un 1,02\% y el de 2012 (sin datos disponibles); y iii) se vislumbra un posible declive (tendencial) de las tasas de variación interanual a lo largo de toda la serie - visibilizado con la línea segmentada de color negro- evidenciada, además, por las tasas promedio para las décadas de los 60 (4,78\%), 70 (3,49\%), 80 (2,30\%), 90 (2,30\%) y del dos mil (1,51\%).

Por el lado de la formación neta de capital fijo (FNKF) es posible detectar, en atención a la misma tabla, ciertas similitudes (gráficas) en relación a la evolución del PIB, por lo tanto, las reflexiones anotadas (en el párrafo anterior) potencialmente son extrapolables. Por otro lado, en atención a los datos examinados se intuye, nuevamente, una dinámica de acumulación contradictoria con crecientes dificultades conforme lo manifiestan las tasas de crecimiento interanual promedio durante las décadas de los 6o (5,96\%), 70 (2,24\%), 80 (2,62\%), 90 (2,54\%) y del dos mil (o,77\%). Adicionalmente, la FNKF parece revelar mayor volatilidad además de un cierto grado de anticipación —en relación con las variaciones del PIB - lo que, a nuestro juicio, podría suponer la medida en que la inversión empresarial ( $\mathrm{FBKF}$ ) guía las pautas del proceso de acumulación (ver Figura 3).

Partiendo de estas observaciones, se pueden apuntar varios hechos suscitados en la economía norteamericana en relación con la evolución del PIB: i) se suscitan periodos de crisis con alguna periodicidad independientemente de su contundencia, ello se manifiesta claramente en 1973-1974, 1980, 1982, 1991 y 2008-2009; ii) los procesos de recuperación de estas crisis se manifiestan con menor intensidad - expresado en la tasa de variación interanual promedio de los tres años de recuperación-, así, el periodo de crisis de 1973-1974 lleva aparejado un 5,23\%, el de 1980 y 1982 un excepcional ${ }^{25} 5,27 \%$, la de 1991 un 3,46\% y la de 2008-2009 un 2,13\%; y iii) existe una bajada tendencial de las tasas de variación interanual a lo largo de toda la serie visibilizado con la línea segmentada en color gris- evidenciado, además, en las tasas promedio de las décadas de los 60 (4,69\%), 70 (3,32\%), 80 (3,04\%), 90 de forma contratendencial ${ }^{26}$ $(3,21 \%)$ y del dos mil $(1,73 \%)$.

Considerando a la formación neta de capital fijo (FNKF) como un vector de inversión, es posible determinar tentativamente, en atención a la misma tabla, ciertos patrones de comportamiento comunes en relación a la evolución del PIB, así las premisas anotadas (párrafo anterior) son, de algún modo, replicables. Del mismo modo atendiendo a los datos se evidencia, 
Figura 2. Evolución de la tasa de acumulación (FNKF/K) (\%), UE(15) y EE. UU.

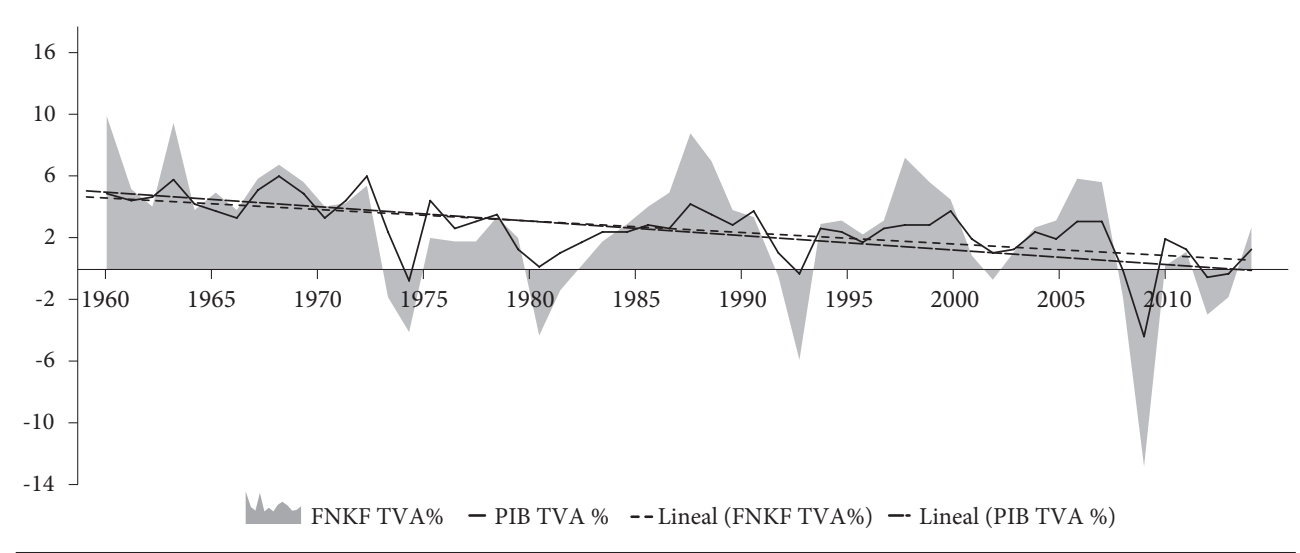

Fuente: Elaboración propia a partir de los datos de AMEco.

Figura 3. Tasa de variación interanual del PIB y de la formación neta de capital fijo (FNKF), EE. UU.

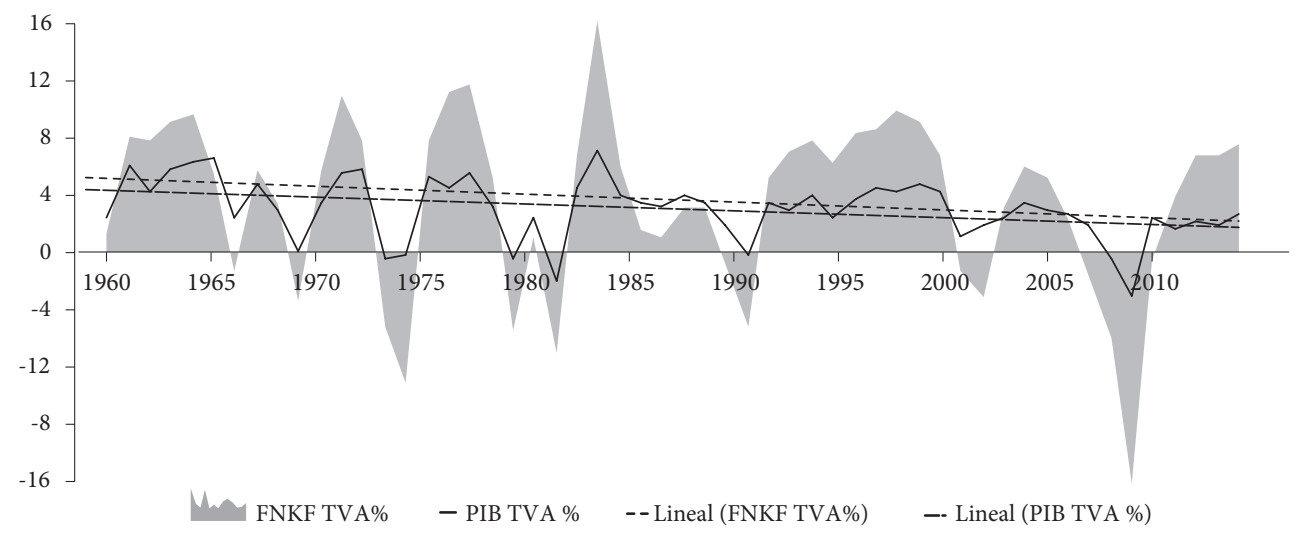

Fuente: Elaboración propia a partir de los datos de Ameco.

igual que en la economía de la UE-15, una posible dinámica de acumulación contradictoria que denota dificultades crecientes. Así lo reflejan las tasas de crecimiento interanual promedio que muestran un tendencial descenso en las décadas de los 60 (5,62\%), 70 (4,41\%), 80 (2,69\%), 90 de forma extraordinaria ${ }^{27}(5,73 \%)$ y del dos mil (-0,36\%). Adicionalmente, la FNKF revela mayor nivel de volatilidad sumado a un cierto grado de anticipación (en relación con las variaciones del PIB) lo que, a nuestro juicio, podría explicar, en alguna medida, que las pautas del proceso de acumulación vienen marcadas por el compás de la inversión empresarial (FBKF). Concluyentemente, es posible apuntar tres elementos comunes que se destacan en ambos bloques económicos: En primera instancia, se constata la presencia de las crisis con una cierta 
regularidad; además, en una segunda instancia, se aprecia el hecho de que el ritmo de acumulación (expresado como un símil de la evolución del PIB y FNKF) tiende a ralentizarse. Adicionalmente, un tercer elemento, sin duda gravitante, parte del hecho de evidenciar la presencia de periodos de crisis cada vez más profundas. Ello se traduce, de alguna manera, en la incapacidad del sistema de producción capitalista para lanzar y relanzar el proceso de acumulación de forma sostenida, no solo por la presencia de crisis con determinada regularidad, sino también debido a procesos de acumulación cada vez con mayores dificultades.

\section{RENTABILIDAD Y TASA DE BENEFICIO}

Una vez revisadas ciertas pautas del proceso de acumulación pasemos al estudio de los componentes de la tasa de beneficios sin perder de vista la premisa del apartado anterior: las economías de la UE-15 y uSA muestran evidencias de su incapacidad para lanzar y relanzar el proceso de acumulación del capital de forma sostenida. Haciendo una breve recapitulación de los postulados marxistas, tenemos que la tasa de ganancia se encuentra definida por: $g^{\prime}=p v /$ $(\mathrm{c}+\mathrm{v})$ o g'=pv'/(1+(c/v)).

Adicionalmente, en el marco de la contabilidad nacional, la tasa de beneficio ${ }^{28}$ se define como: $\Pi=\mathrm{EBE} / \mathrm{K}$, donde $\Pi$ es la tasa de beneficio, EBE es el excedente bruto de explotación o las rentas del capital y $\mathrm{K}$ es el stock de capital y, como se verá más adelante, $\mathrm{L}$ es número de trabajadores. De este modo la tasa de beneficio representa una medida de los beneficios que captan las rentas del capital en relación al conjunto de inversiones que dispone una economía (capital fijo). Siguiendo esta formulación inicial, la tasa de beneficio (П) puede desagregarse de acuerdo a Shaikh (1984) de la siguiente manera:

$$
\Pi=\frac{\frac{E B E}{P I B} \frac{1}{L}}{\frac{K}{P I B} \frac{1}{L}}=\frac{\frac{E B E}{P I B} \frac{P I B}{L}}{\frac{K}{L}} \Rightarrow\left\{\begin{array}{l}
\frac{\partial \Pi}{\partial \frac{E B E}{P I B}}>0 \\
\frac{\partial \Pi}{\partial \frac{P I B}{L}}>0 \\
\frac{\partial \Pi}{\partial \frac{K}{L}}<0
\end{array}\right.
$$

De la expresión anterior se deriva el hecho de que la tasa de beneficio (П) se encuentra compuesta por tres elementos: i) el EBE/PIB que materializa, de algún modo, la lucha de clases o pugna distributiva por la apropiación de las rentas o lo que es su inversa: el salario relativo (RA/ $\mathrm{PIB}$ ); ii) un índice (PIB/L) que refleja una virtual aproximación al concepto de productividad de la fuerza de trabajo; ${ }^{29} \mathrm{y}$ iii) un indicador (K/L) que manifiesta, en alguna medida, el proceso de capitalización del proceso productivo; es decir, el grado o nivel de mecanización de la economía. 
Figura 4. Evolución de la tasa de beneficio (\%), UE(15) y EE. UU.

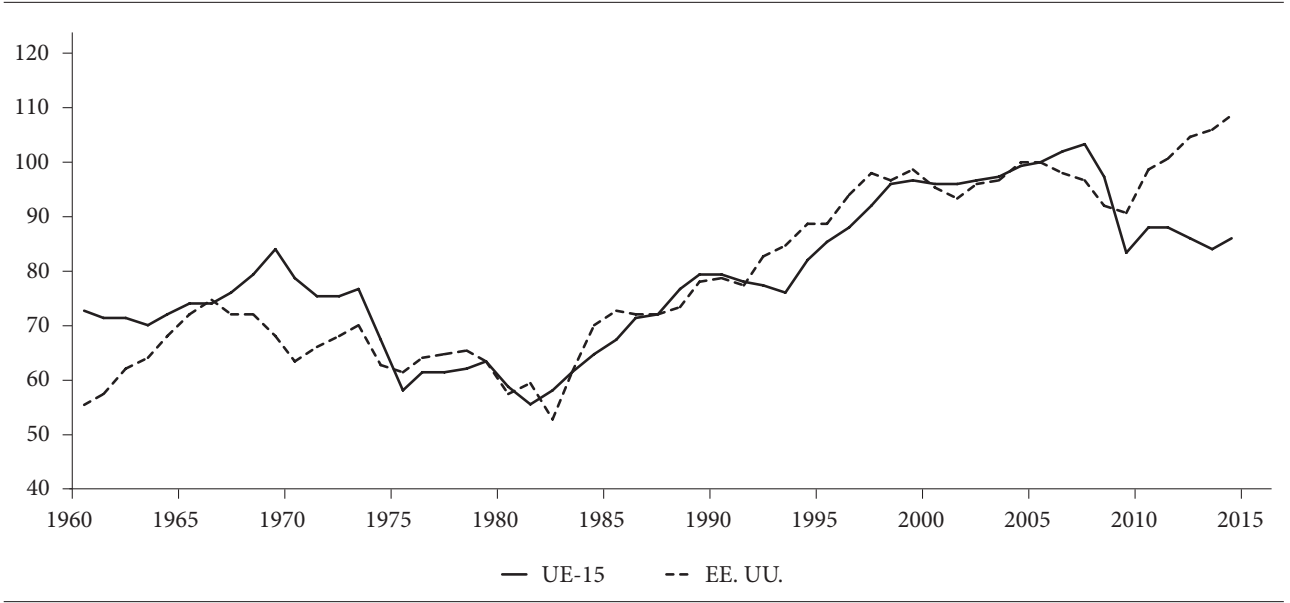

Fuente: Elaboración propia a partir de los datos de AMEco.

Como se aprecia, a través del cálculo derivado, la tasa de beneficio (П) se moverá: i) en el mismo sentido que la pugna distributiva o distribución funcional de la renta favorable al capital (EBE/PIB) y, además, en consonancia con la productividad laboral (PIB/L); y ii) en sentido inverso a la mecanización del proceso productivo $(\mathrm{K} / \mathrm{L})$ o la cantidad de capital requerida por trabajador. Así, diríamos que la evolución de la tasa de beneficio, según la formulación de Marx, responde a la convergencia de estos tres comportamientos de forma simultánea, tal es así que, por ejemplo, una tendencia materializada en un proceso de mecanización creciente (K/L) haría descender la tasa de beneficio (П) siempre y cuando tanto la distribución funcional de la renta (EBE/PIB) como la productividad laboral (PIB/L) de forma conjunta no generen efectos contrarrestantes; es decir, que el aumento de $(\mathrm{K} / \mathrm{L})$ sea mayor que los incrementos agregados de (ЕBE/PIB) y (PIB/L), o dicho formalmente que $\partial П / \partial c / v>\partial П /(\partial \mathrm{EBE} / \mathrm{PIB})$ у $\partial П /$ $(\partial \mathrm{pIB} / \mathrm{L})$. Esta reflexión supondría, por ejemplo, que en un escenario de mecanización creciente, con procesos de aumento de la productividad laboral (en menor medida), requiera necesariamente de ajustes (o presiones) sobre la esfera distributiva a fin de neutralizar una inminente caída de la tasa de beneficio. De este modo, inclusive si el efecto sobre el componente distributivo es lo suficientemente contundente como para anular el aumento neto de la mecanización - es decir, una vez imputado el efecto del incremento de la productividad laboralla tasa de beneficio podría experimentar una evolución en ascenso.

La propuesta global de Marx afirma, en concreto, que es plausible sostener tasas de beneficio en niveles estables (inclusive al alza) en un contexto de mecanización creciente siempre y cuando existan dos condiciones: i) un incremento de la productividad del trabajo; y ii) sobretodo una recomposición (reorganización) de las rentas (cada vez más) favorables hacia el capital. Evidentemente, estas dos fuerzas «contrarrestantes» deben ser cada vez más potentes (ver Figura 4).

En atención a los datos, es posible apuntalar dos indicios: i) el comportamiento de las tasas de beneficio en ambas economías mantiene ciertas características de homogeneidad lo cual 
supone que, en el marco de la economía mundial, ambos bloques, en términos tendenciales, materializan un patrón de sinergias común matizado por los periodos de crisis; y ii) individualmente, a partir de 1960 la economía de la UE-15 mantiene un crecimiento sostenido durante casi una década hasta que de 1970 a 1973 se origina un primer periodo de declive. Por el lado de la economía norteamericana se aprecia, del mismo modo, un periodo de crecimiento cuyo punto de inflexión se sitúa en 1966 que da paso a una caída sostenida hasta la llegada de 1970 a partir de lo cual experimenta una breve recuperación hasta 1973. A partir de este año, ambas economías, en términos generales, experimentan evoluciones sincrónicas donde las tasas de beneficios concretan una caída tendencial hasta los primeros años 80 cuya recuperación es materializada por una oleada de medidas neoliberale ${ }^{30}$ que provee una nueva propulsión durante casi dos décadas según Arrizabalo (2014). A partir de los años dos mil, dichas tasas logran estabilizarse en espera de una nueva crisis mundial que hace su irrupción en 2008-2009.

No obstante, a la par de este análisis surge una primera reflexión que pone de manifiesto una de las contradicciones del sistema capitalista, por una parte - de acuerdo a las premisas inmediatas anteriores - el proceso de evolución de las tasas de beneficio, a partir de los primeros años 80 , denota el restablecimiento de la rentabilidad que se sostiene durante alrededor de tres décadas; sin embargo, este desempeño favorable en ambas economías no ha sido acompañado de un proceso de acumulación sostenido (aludiendo a los apartados anteriores en torno a su incapacidad para autorregenerarse) que, en todo caso, sería lo esperable. Recordemos que, bajo la lógica capitalista, la rentabilidad guía a la inversión y, por tanto, conduce el proceso de acumulación; sin embargo, a la luz de estas contradicciones yace el hecho de que una tasa de beneficios ascendiente, a la par de un proceso de acumulación que cada vez crece menos, remite consecuentemente su sostenibilidad hacia el plano de la disputa distributiva. En otras palabras, desde la década de los 80 hasta la llegada de la crisis de 2008, asistimos a un crecimiento del PIB (cada vez más) ralentizado; es decir, una acumulación cada vez menor - lo cual no significa que haya incrementos cero sino en escala descendiente-; sin embargo, la repartición de ese PIB para los inversores aún genera proporciones crecientes de valorización del capital - tasas de beneficios en aumento; es decir, atractivas-.

\section{ANÁLISIS DE LOS COMPONENTES DE LA TASA DE BENEFICIO}

Según el marco teórico de Marx, en la tasa de beneficio convergen tres vectores: la productividad del trabajo, la composición orgánica del capital y la disputa distributiva por las rentas. Considerando que la tasa de beneficio, a partir de los 80, experimenta un periodo de recuperación sostenida — hasta la llegada de la crisis de 2008-2009 - ${ }^{31}$ a la par de una tasa de acumulación que crece cada vez menos, surgen inmediatamente dos interrogantes: i) ¿cuáles son los elementos que están determinando ese aumento tendencial de la tasa de beneficio?; y ii) ¿en qué medida éstos interactúan provocando el movimiento ascendente de la rentabilidad (tasa de beneficio)? (ver Figura 5).

En vista de las interrogantes planteadas, y en atención a los datos, que muestra la evolución de los componentes de la tasa de beneficio de la economía de la UE-15, se establecen tres indicios: i) un crecimiento moderado de la productividad del trabajo (PIB/L) medido en términos 
Figura 5. Evolución de los componentes de la tasa de beneficio, UE(15)

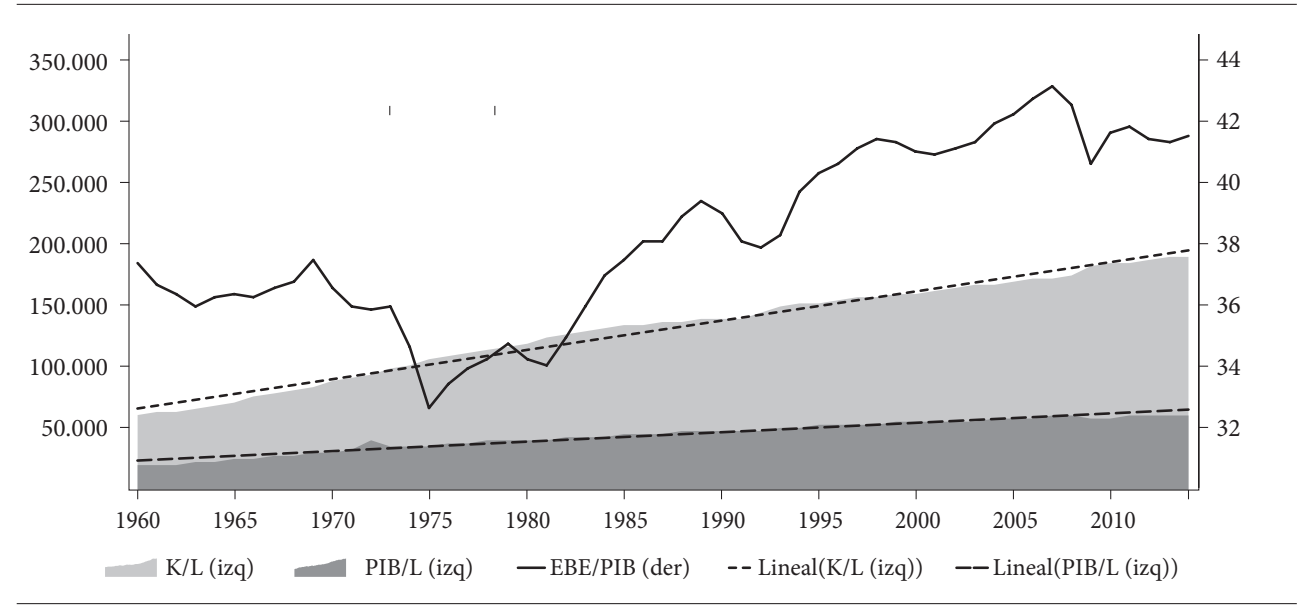

Fuente: Elaboración propia a partir de los datos de AMEco.

Figura 6. Evolución de los componentes de la tasa de beneficio, EE. UU.

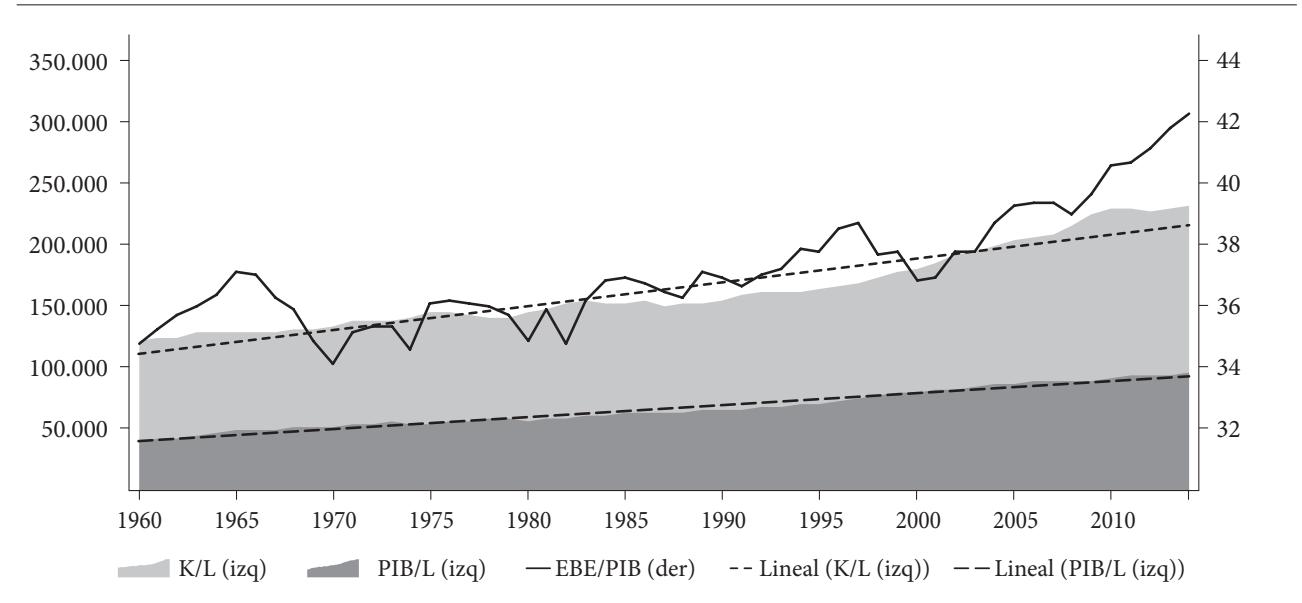

Fuente: Elaboración propia a partir de los datos de AMEco.

de la cantidad de valor producido por trabajador medio; ii) un incremento más acentuado de la composición de capital (K/L) en relación a la productividad laboral; y iii) a partir de las crisis de los 70, un comportamiento tendencial ascendente del peso de los beneficios/rentas del capital en relación con la producción total (EBE/PIB). De los indicios i y ii podemos destacar que el proceso de mecanización de la economía de la UE-15, produce un aumento en la productividad del trabajo en menor proporción que el incremento de la composición orgánica del capital. Ello se puede demostrar analíticamente a través de regresiones lineales simples: 
$\left.\begin{array}{l}y_{1}=2.383\left(x_{1}\right)+64.651 \rightarrow R^{2}=0,985 \rightarrow \frac{\partial y_{1}}{\partial x_{1}}=2.383 \\ y_{2}=776,7\left(x_{2}\right)+23.527 \rightarrow R^{2}=0,972 \rightarrow \frac{\partial y_{2}}{\partial x_{2}}=776,7\end{array}\right\} \Rightarrow \frac{\partial y_{1}}{\partial x_{1}}>\frac{\partial y_{2}}{\partial x_{2}}$

Siendo $\mathrm{y}_{1}$ la variable dependiente $(\mathrm{K} / \mathrm{L})$ y $\mathrm{x}_{1}$ la variable independiente (año) para la composición orgánica de capital. Del mismo modo $\mathrm{y}_{2}$ la variable dependiente estimada (pib/L) y $\mathrm{x}_{2}$ la variable independiente (año) para la productividad del trabajo.

De acuerdo a esta formulación, mediante regresiones lineales simples, se puede deducir que con un alto grado de fiabilidad (R2 superiores a 97\%) el crecimiento del ratio K/L excede al aumento del ratio PIB/L tal como lo demuestra el cálculo derivado. Dicho de otro modo, y de acuerdo con las estimaciones realizadas para la economía de la UE-15, cada año la composición orgánica del capital, en términos tendenciales, aumenta casi el triple del incremento de la productividad del trabajo. En virtud de lo anterior, en un contexto hipotético, si únicamente considerásemos los efectos de estas dos vertientes (к/L y PIB/L), la tasa de beneficio de la UE-15 mantendría una tendencia a la baja. No obstante la verificación este crecimiento, en particular a partir de los primeros años 80 supondría, tentativamente, que este incremento se dilucida fundamentalmente en el ámbito de la pugna distributiva que es corroborado, además, por la tasa interanual promedio de crecimiento de las rentas del capital (EBE/PIB) que asciende a o,60\% durante las tres últimas décadas (ver Figura 6).

En concordancia con lo anterior, se establece, de forma muy parecida al caso de los países de la UE-15, tres premisas para la economía norteamericana: i) un crecimiento medianamente moderado de la productividad del trabajo (PIB/L); ii) un incremento más contundente de la composición de capital (K/L) en relación a la productividad laboral; y iii) a partir de los años 70, un comportamiento tendencial ascendente del peso de los beneficios favorables al capital en relación con la producción total (EBE/PIB). De las premisas i y ii podemos subrayar que el proceso de mecanización de la economía norteamericana produce, paralelamente, un aumento en la productividad del trabajo en menor proporción que el incremento de la composición orgánica del capital. Ello se puede demostrar analíticamente a través de regresiones lineales simples:

$$
\left.\begin{array}{l}
y_{1}=1.944\left(x_{1}\right)+10.932 \rightarrow R^{2}=0,902 \rightarrow \frac{\partial y_{1}}{\partial x_{1}}=1.944 \\
y_{2}=978,2\left(x_{2}\right)+39.181 \rightarrow R^{2}=0,974 \rightarrow \frac{\partial y_{2}}{\partial x_{2}}=978,2
\end{array}\right\} \Rightarrow \frac{\partial y_{1}}{\partial x_{1}}>\frac{\partial y_{2}}{\partial x_{2}}
$$

Siendo $\mathrm{y}_{1}$ la variable dependiente $(\mathrm{K} / \mathrm{L})$ y $\mathrm{x}_{1}$ la variable independiente (año) para la composición orgánica de capital. Del mismo modo $\mathrm{y}_{2}$ la variable dependiente estimada (pib/L) y $\mathrm{x}_{2}$ la variable independiente (año) para la productividad del trabajo.

De acuerdo con esta formulación se puede deducir que, con un grado de fiabilidad superior al 90\% (R2), el crecimiento del ratio K/L excede al aumento del ratio PIB/L tal como lo demuestra 
el cálculo derivado. Dicho de otro modo, y de acuerdo con las estimaciones para la economía norteamericana, cada año la composición orgánica del capital, en términos tendenciales, aumenta aproximadamente el doble del incremento de la productividad del trabajo.

Del mismo modo que para la economía de la UE-15, en virtud de lo anterior, en un contexto hipotético, si únicamente considerásemos los efectos de estas dos vertientes (K/L y PIB/L), la tasa de beneficio de la economía norteamericana mantendría una tendencia decreciente. No obstante, la verificación de su crecimiento a partir de los primeros años 80 supondría, tentativamente, que este ascenso se dilucida fundamentalmente en base al ámbito de la pugna distributiva, mismo que se corrobora, además, con el crecimiento de las rentas de capital (EBE/PIB) a una tasa interanual promedio de $0,43 \%$ durante las tres últimas décadas.

Al examinar la evolución de ambas economías, y en concordancia con lo que sostienen Guerrero (2006), Mateo (2007) y Cámara y Flores (2010), asumiendo que en la actualidad asistimos a un creciente proceso de mecanización de la economía, que potencialmente genera un efecto de sobreacumulación, deberíamos contemplar la posibilidad de un permanente colapso generalizado de la tasa de beneficio; sin embargo, ello no ocurre tal como lo verifican los datos. Lo que nos remite nuevamente a considerar, en base a las deducciones anteriores, que la sostenibilidad de la rentabilidad - particularmente explicada mediante la tasa de beneficio- tentativamente se disputaría a través de las fuerzas contrarrestantes, esencialmente las que se remiten al plano de las relaciones de distribución de la renta entre los capitales (capitalistas) y la fuerza de trabajo (trabajadores).

\section{UN ACERCAMIENTO A LA IDEA LUCHA DE CLASES}

Generalizadamente, uno de los aspectos que tiende a soslayarse, y que intencionalmente destacamos para complementar las aseveraciones contenidas en el apartado anterior (específicamente respecto de la pugna distributiva), es la dinámica de lucha de clases que acontece inmersa en el proceso productivo, donde se produce un primer acto de antagonismo entre el capitalista (inversor o, de manera más afinada, poseedor de los medios de producción) y el obrero (trabajador asalariado). Este aspecto fue considerado por Marx, quien, según afirma Mandel (1976) «integra lógicamente el desarrollo de la lucha de clases entre capital y trabajo a su análisis de la producción del plusvalor, en la medida en que considera que la lucha de clases se origina en ese proceso de producción» (p. 32). En atención de lo anterior se propone considerar como indicadores de la pugna de clases a: i) la distribución funcional de la renta; y ii) el dividendo de las rentas del capital en relación con las remuneraciones de los asalariados (proxy de tasa de plusvalía).

Por un lado (literal i), la distribución funcional de la renta [RA/PIB] o dividendo de las rentas de acuerdo con la función material de los actores del proceso productivo (a saber, capitalistas y asalariados), refleja la fracción de las retribuciones de la renta que se asigna a la clase trabajadora y que se manifiesta a través de la masa de remuneraciones de los asalariados (RA $)^{32}$ en relación al valor total creado (РІВ). En términos de categorías marxistas la distribución funcional de la renta refleja un proxy de la fracción v/(c+v+pv) (ver Figura 7$)$.

Así los datos de los países de la Unión Europea (15), muestran el peso relativo de los salarios en torno al conjunto de la producción, donde se aprecia un primer periodo de relativa estabilidad 
entre los años 1960 a 1973 con una tasa que oscila en torno al 63,5\%. A partir de los años 8o, durante tres décadas, se evidencia, en cambio, una caída tendencial que supone una tasa de variación interanual promedio de -0,35\% (o acumulada de -10.94\%). O dicho de otra manera, en el periodo 1980-2010 la proporción de los salarios pierde 7,34 puntos porcentuales.

De manera similar, aunque con una caída menos marcada la economía norteamericana experimenta un decrecimiento tendencial del peso de los salarios respecto al PIB. No obstante, nos llama la atención, sobre todo el periodo desde los 80 hasta los dos mil, que muestra un desplome sostenido cuya la tasa de variación interanual promedio es de -0,25\% (o acumulada de -7,72\%). O dicho de otro modo, para el periodo 1980-2010, el peso de los salarios respecto al PIB norteamericano retrocede 5,69 puntos porcentuales. En apego a estos dos resultados, se evidencia tentativamente un patrón evolutivo común: ambas economías experimentan un tendiente retroceso de la masa salarial en relación al PIB (ver líneas punteadas en Tabla 7).

Por otro lado (literal ii) el dividendo de las rentas del capital en relación con la remuneración de los asalariados [EBE/RA] que sintetiza la propuesta de un índice que relaciona el excedente bruto de explotación (EBE), como una medida aproximada de la masa de plusvalía; es decir, del valor nuevo creado expropiado a la fuerza de trabajo, o simplemente el beneficio capitalista, en relación con la masa de remuneración de asalariados (RA), como un símil del capital variable; constituye un acercamiento a la categoría de tasa de plusvalía (pv') propuesta por Marx. Así, el índice EBE/RA es congruentemente consistente con la formulación de la tasa de plusvalía $\mathrm{pv}=\mathrm{pv} / \mathrm{v}$; es decir, EBE/RA $\approx$ pv'. Otra forma de ver esta relación de acuerdo a la propuesta de Shaik (1984) sería:

$\Pi=\frac{\frac{E B E}{P I B} * \frac{P I B}{L}}{\frac{K}{L}}=\frac{\frac{P I B-R A}{P I B} * \frac{P I B}{L}}{\frac{K}{L}}=\frac{\frac{P I B-R A}{L}}{\frac{K}{L}}=\frac{\overbrace{P I B}^{L}-\frac{R A}{L}}{\frac{K}{L}} \approx \frac{\overbrace{p v^{\prime}}^{b}}{1+\frac{c}{v}} \approx g^{\prime}$

Teóricamente, remitiéndonos a la fórmula 6, diríamos que en a se representan (por trabajador): en $\mathrm{PIB} / \mathrm{L}$ la cantidad de valor creado en el proceso productivo, mientras en RA/L el valor pagado/ sufragado (a la fuerza de trabajo); el valor creado menos el valor pagado constituye el principio del valor expropiado al trabajador, el cual concreta, según Marx, a la masa de plusvalía que, expresada de otra manera, constituye la tasa de plusvalía pv' contenida en b. Empíricamente, el ratio EBE/RA se aproxima, de algún modo, la relación de las rentas apropiadas por el capital respecto de las rentas de los asalariados — principio constitutivo de la tasa de plusvalía- (ver Figura 8).

Se constata, en atención de lo anterior, que en los países de la Unión Europea (15), el peso de los beneficios de las rentas del capital en relación con las rentas de los asalariados evidencia dos comportamientos plenamente identificados. En las décadas de los 60, 70 y 80 el ratio EBE/RA decrece en torno a una tasa de variación interanual promedio de -1,63\%. No obstante, a partir de los años 80 estas tasas muestra señales de recuperación que se sostienen tendencialmente durante tres décadas mostrando una la tasa de variación promedio interanual de 0,53\% exceptuando una fuerte caída para el periodo de 1990 a 1992 (donde la tasa de variación interanual promedio se sitúa en -2,44\%) y entre 2008 y 2009 (donde ésta llega a -4,10\%). 
Figura 7. Remuneración asalariados en proporción al PIB o salario efectivo (\%), UE (15) y EE. UU.

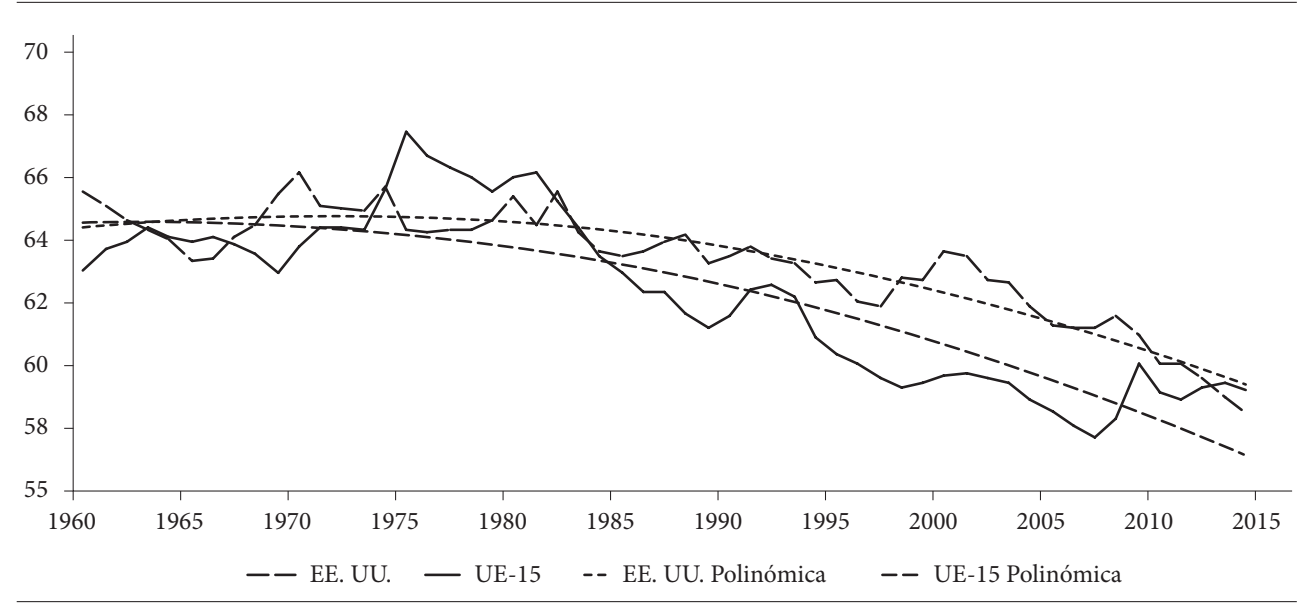

Fuente: Elaboración propia a partir de los datos de AMEco.

Figura 8. Relación dividendo de rentas de capital y remuneración asalariados (\%), EE. UU. y UE (15)

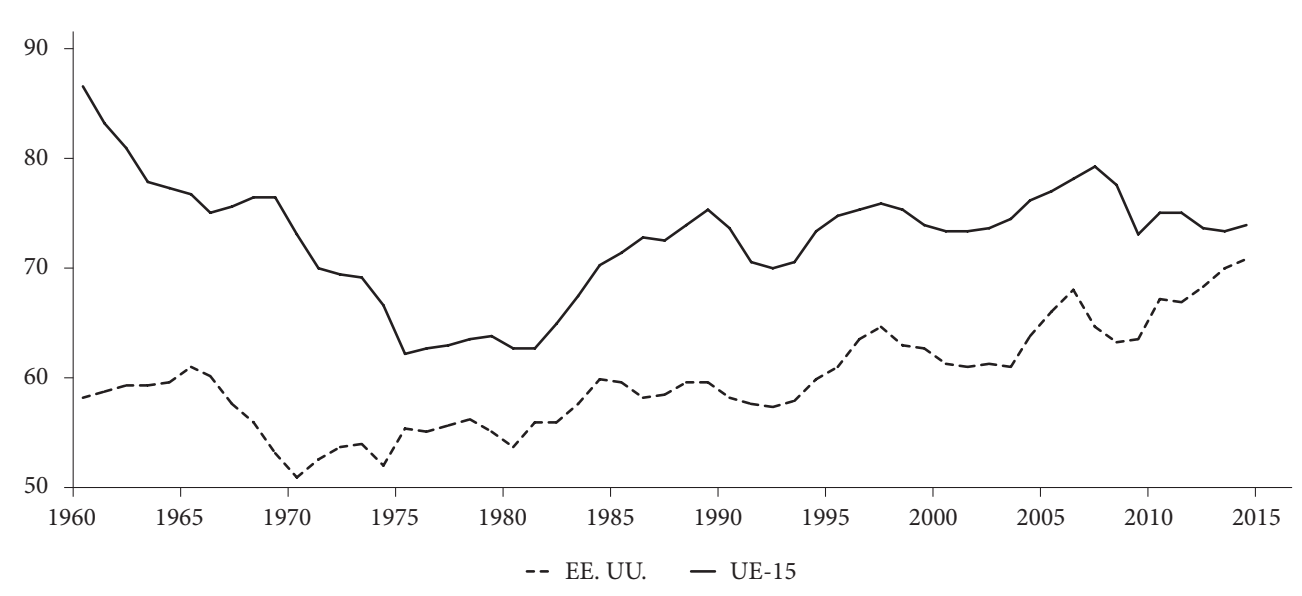

Fuente: Elaboración propia a partir de los datos de AMEco.

Asimismo, podemos destacar que en la economía norteamericana el peso de los beneficios de las rentas del capital en relación con las rentas de los asalariados evidencia dos grandes fases. La primera con un marcado ascenso entre 1960 y 1965 (tasa de variación interanual promedio de 1,01\%) y a partir de ello una caída precipitosa hasta 1970 con una tasa de variación interanual promedio de $-3,66 \%$ como consecuencia de la crisis económica acontecida en los años 70 cuyo resurgimiento, en términos generales, se materializa mediante la adopción de políticas neoliberales cuyos íconos encarnan las figuras del presidente Ronald Reagan (Estados Unidos) y la Primera Ministra Margaret Thatcher (Reino Unido) siguiendo las tesis de Arrizabalo (2014). 
De esta manera se logra reconfigurar la proporción EBE/RA permitiendo un crecimiento sostenido cuya tasa de variación interanual promedio se sitúa en $0,87 \%$ que está presente desde 1971 a 2006, para finalmente en 2007 y 2008 experimentar una caída en torno a una tasa de variación interanual de $-3,57 \%$ producto de una nueva crisis económica.

En resumen se verifica que, para el caso de ambas economías, tanto la evolución de la distribución funcional de la renta como el comportamiento de dividendo de las rentas del capital (en relación con la remuneración de los asalariados), evidencian cualitativamente el entramado de tensiones crecientes (pugnas distributivas) por la apropiación del excedente que (a partir de 1971 para EE. UU. y 1975 para UE-15) favorece habitualmente al capital.

A pesar de que en los apartados anteriores hemos abordado rápidamente algunas referencias empíricas que caracterizan la presencia de los periodos de crisis, resulta fundamental partir del hecho de que una tendencia decreciente de la tasa de beneficios puede constituirse como el principal factor que explicaría el cortocircuito del proceso de acumulación. La presencia, por tanto, de periodos de crisis supone, implícitamente, contratendencias insuficientes (ver Figuras 9 y 10).

Para ilustrar conjuntamente el comportamiento de la tasa de beneficio (medida de rentabilidad), el proceso de acumulación (medida de regeneración) y el crecimiento del PIв (medida de evolución productiva) acudimos a sus correspondientes índices (ver Figuras 9 y 10). Aquí parece esclarecerse un patrón común en ambas economías que supondría dos planteamientos: i) en la medida en la que la tasa de beneficio sufre decrecimientos considerables, la tasa de variación del PIB cae, tal como lo demuestra, para el caso de los países de la UE-15, los años de 1975, 1981, 1993 y 2009, así como para el caso de la economía norteamericana los años 1970, 1975, 1980, 1982, 1991, 2001 y 2009; y ii) menos acentuadamente el decrecimiento de la tasa de beneficio guarda relación con la tasa de acumulación tal como lo demuestran los años 1975, 1981, 1993 y 2009 para los países de la UE-15, así como en los años 1970, 1975, 1982, 1991, 2001 y 2010 para la economía norteamericana. Es fundamental esclarecer que la evolución de estas variables (tasa de beneficio, tasa de acumulación y evolución del PIB) muestra sus niveles de correlación más no necesariamente sus relaciones de causalidad (ver Figura 11).

Recapitulemos de manera esquemática la serie de relaciones que entre los indicadores se han venido mencionando a lo largo del el tratamiento empírico (ver Figura 1). Así, en el sistema de producción capitalista, la lucha de clases constituye el eje vertebrador sobre la base del cual se dilucida la disputa distributiva; ${ }^{33}$ es decir, la concreción del reparto de las rentas que van a manos de los asalariados o de los capitalistas. La disputa por la distribución de estas rentas es el primero de los tres componentes que inciden de manera directa en la tasa de beneficio. A continuación, es necesario clarificar el hecho de que la tasa de beneficio va a marcar el ritmo de acumulación del proceso productivo que, sinergialmente, impacta sobre el nivel de mecanización de la economía o aumento en la composición orgánica del capital (segundo componente) y que, a su vez, genera un efecto sobre la productividad del trabajo. Ésta materializa el nivel de eficiencia laboral (tercer componente) que afecta, consecuentemente, a la tasa de beneficio. Por otro lado, como hemos explicado, un incremento en la tasa de beneficio tira del ritmode acumulación que, de forma simultánea, origina tensiones crecientes para la mecanización del proceso productivo que, a pesar de que incide positivamente sobre la productividad del trabajo (en un 
Figura 9. Tasa de beneficio, tasa de acumulación y tasa de variación interanual del PIB, UE-15

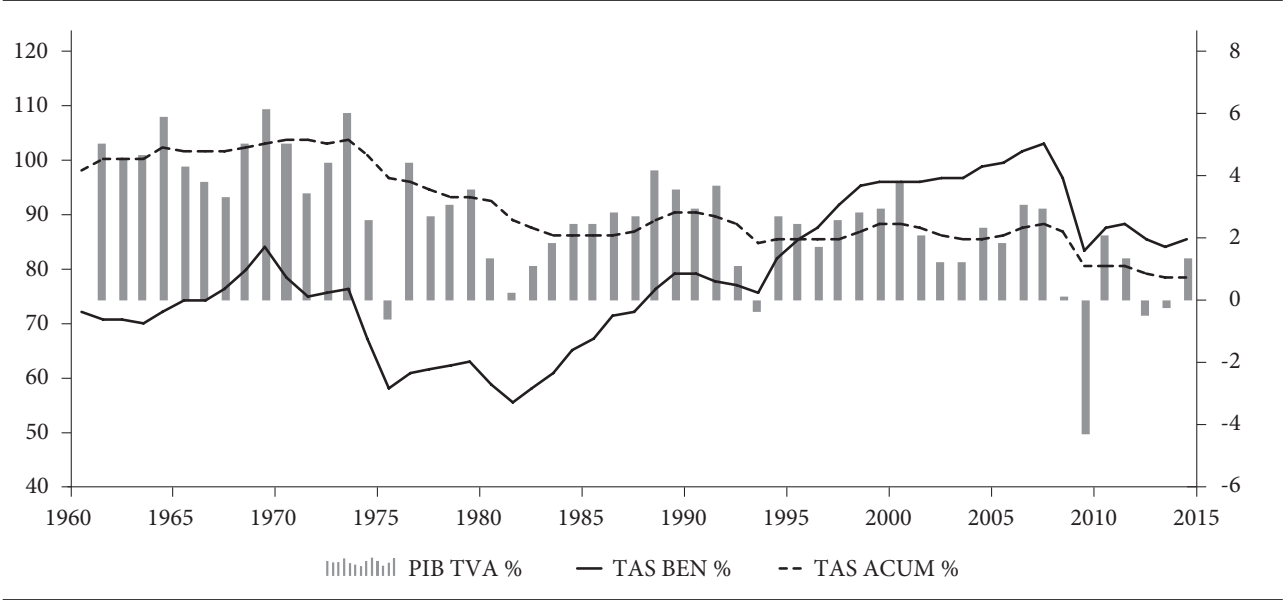

Fuente: Elaboración propia a partir de los datos de AMEco.

Figura 10. Tasa de beneficio, tasa de acumulación y tasa de variación interanual del PIB, EE. UU.

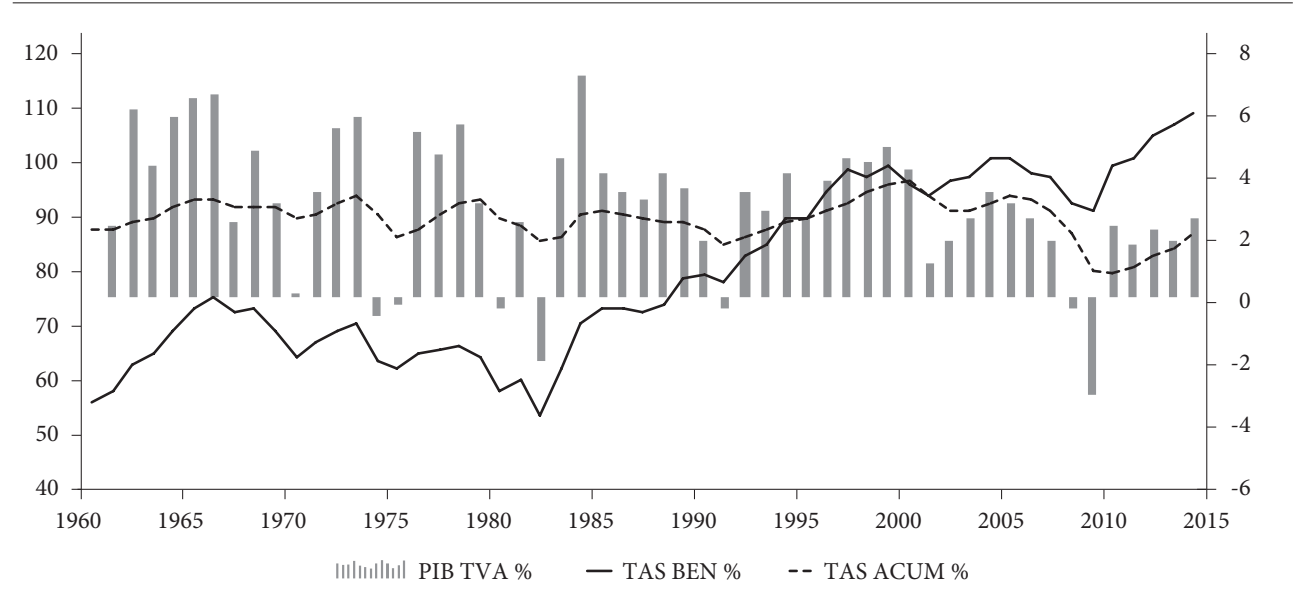

Fuente: Elaboración propia a partir de los datos de AMEco.

grado menor al proporcional), en última instancia, instaura presiones para el descenso de la tasa de beneficio que, acompañado por la incapacidad para reestructurar la pugna distributiva (lucha de clases) en favor de las rentas del capital, desemboca, en definitiva, en los procesos de crisis.

\section{CONCLUSIONES}

La primera deducción (con un carácter introductorio) extensible al resto de conclusiones parte de la dificultad al transpolar linealmente la información contenida en el Sistema de Cuentas 
Nacionales hacia el ámbito del pensamiento abstracto de Marx como consecuencia de las distintas fundamentaciones metódicas a las que responden ambas propuestas analíticas; esto constituye, por sí mismo, un limitante a ser considerado. No obstante, el aporte teórico marxista, concretamente la Ley del Descenso Tendencial de la Tasa de Ganancia, observado desde las estadísticas convencionales puras $^{34}$ proporciona, sin duda, una matriz teórico-conceptual suficientemente potente a la hora de interpretar ciertas correlaciones estructurales (capital-trabajo) insertas en la lógica que opera el sistema de producción capitalista.

Primero, el ritmo de acumulación, en términos generales, concebido desde la evolución de la tasa de acumulación, que relaciona la inversión (FNKF) y el stock de capital, evidencia muestras de agotamiento (ralentización), lo cual no significa que llegue necesariamente a cero, sino que cada vez es menos intenso. Ello supone la presencia de ciertas dificultades en el proceso de acumulación por lo que se clarifica, de alguna manera, la incapacidad del sistema de producción capitalista para lanzar y relanzar el proceso de acumulación de forma sostenida no solo por la presencia de crisis recurrentes - cada vez más contundentes y con periodos de recuperación de menor intensidad-, sino también debido a tasas de acumulación cada vez menores.

Segundo, la evolución de las tasas de beneficio permiten esclarecer, en una primera instancia, un comportamiento tendencial homogéneo en ambos bloques económicos lo cual supone, en el marco de la economía mundial, un patrón de sinergias que se va matizando por la presencia de periodos de crisis. Así se confirma que en la década de los 60 los países de la UE-15 mantienen un crecimiento sostenido hasta que de 1970 a 1973 se origina un primer periodo de declive. Por el lado del bloque norteamericano se aprecia, del mismo modo, un primer periodo de crecimiento cuyo punto de inflexión se sitúa en 1966 que da paso a una caída hasta la llegada de 1970 a partir de lo cual experimenta una breve recuperación hasta 1973. A partir de este año, ambas economías, en términos tendenciales, experimentan evoluciones sincrónicas donde las tasas de beneficios concretan una caída tendencial hasta los primeros años 8o, cuya recuperación se sostiene durante casi dos décadas de forma ininterrumpida. A partir de los años dos mil, estas tasas continúan con un crecimiento más sutil hasta la irrupción de la nueva crisis mundial que hace su aparición en 2008.

Bajo una perspectiva de largo alcance, en segunda instancia, es posible afirmar que las tasas de beneficio en ambos bloques económicos experimentan (mayoritariamente) un crecimiento tendencial en las décadas de los años 60, 80, 90 y dos mil, con excepción del periodo de la década de los 70. De este modo, podemos intuir que las tasas de beneficio mantienen un crecimiento generalizado que se interrumpe con la llegada de la crisis de los años 70 y, posteriormente, con la crisis de 2008. Ello nos lleva a ratificar que la tasa de beneficio en términos generales (proxy de la tasa de ganancia) tiende de a crecer ${ }^{35}$ lo cual supone, de acuerdo al planteamiento de Marx, la coexistencia de un conjunto de fuerzas contrarrestantes.

Tercero, en atención a los componentes de la tasa de beneficio, se establecen varios indicios que apuntan en dirección a: i) un incremento (acentuado) de la composición de capital $(\mathrm{K} / \mathrm{L})$ en relación a la productividad laboral; ii) un crecimiento (moderado) de la productividad del trabajo ( $\mathrm{PIB} / \mathrm{L}$ ) medido en términos de la cantidad de valor producido por trabajador medio; y iii) en lo relativo al peso de los beneficios del capital en relación con la producción total (EBE/PIB): un moderado descenso en la primera mitad de los 60 seguido por un 
Figura 11. Esquema de la tasa de beneficio

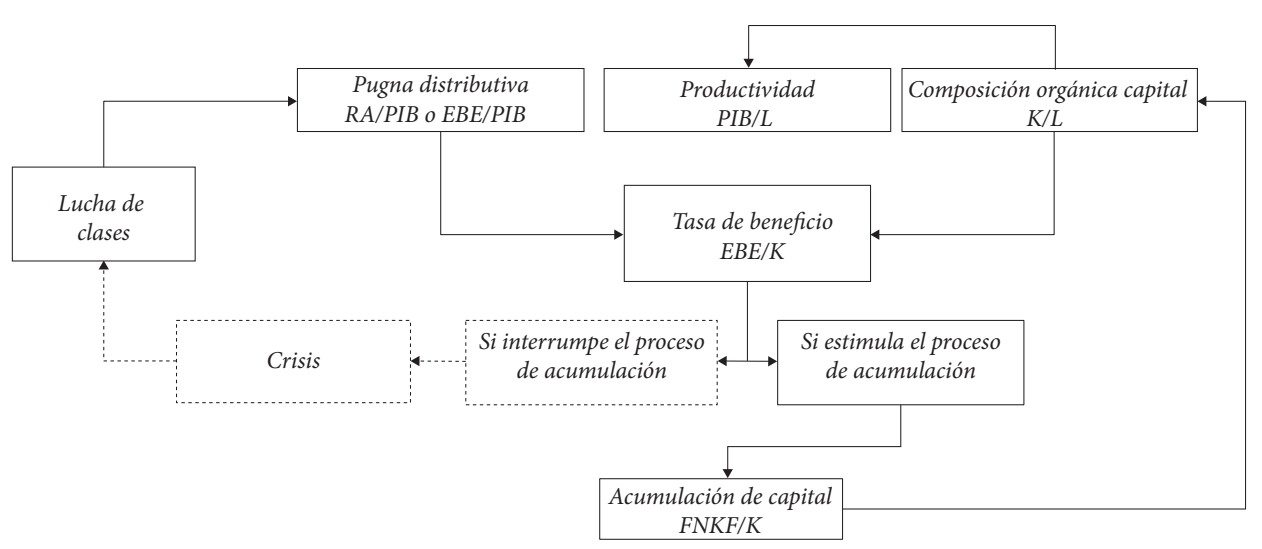

Fuente: Elaboración propia.

periodo de recuperación hasta la llegada de los 70 para el bloque de la UE-15; así como un ascenso más pronunciado durante la primera mitad de los 60 para, después, marcar una caída importante durante la segunda mitad para el bloque norteamericano. Luego, ambas economías se encuentran afectadas por la llegada crisis de los 70, cuyos puntos de inflexión (1971 para USA y 1975 para la UE-15) recomponen las condiciones distributivas (en pugna) favoreciendo las alícuotas de los capitales concretando así un ascenso sostenido hasta la llegada de la nueva crisis en 2008. De las premisas i y ii podemos destacar que el proceso de mecanización de la economía, como efecto de las inversiones realizadas por el capital - fruto ente otras cosas de un contexto crecientemente competitivo- produce derivadamente un aumento en la productividad del trabajo en menor proporción que el incremento de la composición orgánica del capital; es decir, que el flujo de inversiones realizadas, en términos de capital por trabajador, no genera, en la misma proporción, un aumento de la productividad del trabajo. En virtud de ello, si consideramos el incremento acentuado de la composición del capital y el aumento, en menor medida, de la productividad laboral, y lo añadimos al descenso de la proporción de las rentas del capital respecto del PIB (especialmente en los periodos 1965-1970 USA y 1970-1975 UE-15), las tasas de beneficio concretan caídas reales - perfectamente explicables en el contexto de la crisis de los 70-. No obstante, la verificación del crecimiento de la tasa de beneficio a partir de los 80 , que marca la salida de la crisis de los años 70, supone que dicha recuperación se encuentra soportada fundamentalmente en el ámbito de la pugna distributiva que se corrobora a través de un acentuado crecimiento de las rentas favorables al capital (en relación a la rentas de los asalariados) a partir de 1971 para USA y 1975 para UE-15. Adicionalmente, a través de la evolución de la tasa de beneficio, es posible abordar metodológicamente una posible explicación sobre los periodos de crisis. Esta premisa implica, por lo pronto, descartar tácitamente el hecho de que la génesis de las crisis descansa sobre una serie de elementos exógenos a la propia dinámica de acumulación capitalista. Así, es previsible 
considerar que las causas determinantes de las crisis económicas se sitúan en el ámbito (mismo) de la producción cuando una economía no es capaz de generar o, mejor, regenerar valores nuevos suficientes con la intensidad requerida por el capital.

Cuarto, a la par del planteamiento anterior, excluyendo los periodos de caída de la tasa de beneficio; es decir, considerando el restablecimiento de la rentabilidad (luego de la crisis de los años 70 y 2008), se esclarece el hecho de que este desempeño favorable, para el caso de ambas economías, no va acompañado de la recuperación del proceso de acumulación aludiendo a la incapacidad del sistema de producción para su autorregeneración visible en la caída tendencial de la tasa de acumulación [Conclusión 1] - . Recordemos que, bajo la lógica capitalista, la rentabilidad guía a la inversión y, por tanto, conduce el proceso de acumulación; sin embargo, a la luz de estas contradicciones yace el hecho de que una tasa de beneficios creciente [Conclusión 2], a la par de un proceso de acumulación que cada vez crece pero menos, remite su sostenibilidad al plano de la pugna distributiva. En palabras más simples, los datos demuestran que, desde los 80 hasta la llegada de la crisis de 2008, asistimos a un crecimiento de la tarta (PIB) cada vez más ralentizado; es decir, una acumulación cada vez menor - lo cual no significa que haya incrementos cero, sino en escala descendiente-; sin embargo, la repartición de la tajada para los inversores aún generaría procesos crecientes de valoración del capital — tasas de beneficios en aumento; es decir, atractivas-. Surge, en este punto, un primer indicio que supondría la constatación empírica de una de las contradicciones inmanentes del sistema capitalista.

Quinto, la tensión del conflicto entre trabajo y capital (que menciona Marx) que, se aproxima a la dimensión de la pugna distributiva, se manifiesta empíricamente en dos dimensiones: i) un decremento de la distribución funcional de la renta (RA/PIB) o salario relativo con tasas de variación interanual promedio negativas de, aproximadamente, un tercio de punto (UE-15) y un cuarto de punto (USA); y ii) el aumento del dividendo de las rentas del capital en relación con la remuneración de los asalariados (EBE/RA), como un símil de la plusvalía, con tasas de variación interanual promedio de casi medio punto en los países de la UE-15 y tres cuartos de punto en la economía norteamericana.

Finalmente, la verificación empírica del ascenso de la tasa de beneficio en las economías de los países de la UE-15 y de los Estados Unidos de Norteamérica, durante el último período intercrisis, que involucra un incremento de la mecanización del proceso productivo acompañado por un aumento en la productividad del trabajo (en menor proporción) [Conclusión 3], a la par de un proceso de acumulación ralentizado [Conclusión 1], únicamente puede materializarse a través de un (mayor) aumento de las rentas apropiadas por el capital o, lo que es lo mismo, una disminución del salario relativo [Conclusión 3]. Todo ello apunta a ciertos elementos que nos acercan a la fundamentación abstracta de la propuesta teórica de Marx contenida en la Ley del Descenso Tendencial de la Tasa de Ganancia. De este modo, a nuestro criterio, aunque de forma aún muy acotada, ${ }^{36}$ es posible asumir las reflexiones citadas a lo largo de esta investigación que, evidentemente, no agotan discusiones ni posibles controversias; sin embargo, abren paso a futuras y siempre necesarias profundizaciones. 


\section{NOTAS}

1 La Ley del Valor planteada por Marx alude a un principio normativo de la ciencia económica respecto del intercambio de los bienes y servicios producto de la acción humana. Según Marx (1859), la relación de valores de intercambio de los productos, por lo general expresada por su precio en dinero, son proporcionales a las cantidades de trabajo humano medido en el tiempo gastado, llamado el tiempo de trabajo socialmente necesario para su producción.

2 Si se desea profundizar este aspecto se recomienda la lectura de Guerrero (1997). Un Marx imposible: el marxismo sin teoría laboral del valor.

3 Al respecto, Guerrero (2006, p. 7) sostiene: «Los economistas de la Universidad vienen luego a legitimar de hecho, consciente o inconscientemente, esta manera de ocultar la realidad de la explotación. Para ello afirman que los salarios reales crecen, o que ya no hay auténticos proletarios sino cada vez más clase media. Olvidan que la explotación tiene que ver con la evolución del salario relativo (la participación de los asalariados en la renta nacional, y no con el salario real».

4 Véanse trabajos de Guerrero (1989), Moseley (1991), Duménil y Levy (1993), Shaikh y Tonak (1994), Cámara (2003), Tomé (2006), Maito (2014), entre otros.

5 En otras palabras, la relación entre la fracción de capital contante (medios de producción) y la fracción de capital variable (fuerza de trabajo).

6 Al referirnos al concepto general de tasa de ganancia, nos remitimos a la sesión III de El Capital. Crítica de la Economía Política (1894). Libro III: El Proceso Global de la Producción Capitalista. Madrid: Akal, 1978.

7 A fin de esquematizar con mayor amplitud la dinámica del Proceso Productivo tengamos presente: Producción total $=$ Medios de Producción + Fuerza de Trabajo + Excedente. A partir de lo cual: Excedente $=$ Cuota de acumulación (reinversión) + Consumo improductivo.

8 Al respecto, Tomé (2006, p. 9) afirma: «La tendencia hacia la mecanización progresiva del proceso productivo se funda en la especificidad de la producción capitalista como proceso de valorización o maximización de la rentabilidad del capital».

9 Para ilustrarlo con un símil: al contemplar el vuelo de un aeroplano ¿quién podría defender la idea de que la gravedad no opera? Claro, no es que esta fuerza (gravedad) no actúe, sino que existen contrafuerzas que hacen que el aeroplano se eleve.

10 Además, Marx (1894) apunta, también, otras fuerzas contrarrestantes; por ejemplo: invenciones, aceleración de rotación del capital e inversiones en ramas de baja mecanización.

11 Añadiendo a este conjunto de condiciones la presión de la abundancia del trabajo asalariado que, lógicamente, posibilita un mayor grado de explotación.

12 Suponiendo que el salario real no aumenta y la jornada laboral no disminuye, o, si lo hacen, proporcionalmente en menor medida que el aumento de la productividad.

$13 \mathrm{Al}$ respecto recomendamos los trabajos de Katz (2002) y Mateo (2007).

14 Pues esta no ha sido nuestra pretensión; sin embargo, véanse muy importantes análisis en los trabajos de Rubin (1923) y Robles (2005).

15 Nótese la diferenciación de nomenclatura entre la tasa de ganancia (propuesta por Marx) y la tasa de beneficio (calculada en función de las cuentas nacionales).

16 En estricto rigor, no es capitalización de la economía, sino aumento relativo del capital constante o como lo llamaremos: proceso de mecanización.

17 Creemos importante comentar (en esta parte) el carácter no neutral del Sistema de Cuentas Nacionales por cuanto su misma configuración genética responde, en última instancia, a presupuestos teóricos que marcan definitivamente la perspectiva de análisis; por ejemplo, el ámbito de creación del valor/riqueza, su pertenencia, retribución y distribución es absolutamente esclarecedor: el capital crea valor y, por tanto, es fuente generadora de riqueza. Muy al contrario, siguiendo la propuesta marxista, la única fuente que contiene la capacidad creadora de valor (nuevo) es el trabajo humano. Ambas propuestas teóricas tienen su propia intencionalidad. A nuestro criterio, un ejercicio de honestidad intelectual 
pretendería, al menos, reconocer explícitamente la no neutralidad de la base teórica sobre la cual se reproducen los posteriores desarrollos conceptuales. Por lo tanto, la supuesta neutralidad de Contabilidad Nacional (convencional) supondría, a nuestro entender, un error de partida.

18 Para mayor profundización consúltese a Guerrero (2006).

19 Término utilizado para referirnos al Sistema de Cuentas Nacionales.

20 Denominado también como dividendo de las rentas del capital en relación con la remuneración de los asalariados.

$21 \mathrm{Al}$ respecto, Mateo $(2007$, p. 18$)$ en su análisis sobre la tasa de ganancia de la economía mexicana, cita: «Las categorías esenciales a estimar son el plusvalor [...]. Su cuantificación requiere una modificación del Sistema de Cuentas Nacionales de México (SCNM) para adaptarlo a los postulados de la teoría laboral del valor, lo que agrava las dificultades del trabajo empírico».

22 Ameco es la base de datos macroeconómicos de la Dirección General de la Comisión Europea para Asuntos Económicos y Financieros (DG ECFIN por sus siglas en inglés). Recuperado de http://ec.europa. eu/economy_finance/db_indicators/ameco/

23 Este apartado prevé estudiar la evolución del proceso de acumulación del capital en virtud de que su análisis aporta algunos elementos introductorios claves que servirán al momento de abordar las relaciones de los componentes de la tasa de beneficio.

24 A efectos de dotar de un marco de comparabilidad, se considera dichas variables en términos de tasas de variación interanual.

25 Se ha utilizado la expresión excepcional en virtud del comportamiento de la tasa de variación interanual registrada en 1984 que desdibuja la tendencia y que eleva la tasa de variación interanual promedio de los tres años de recuperación. No obstante, es primordial considerar al conjunto de los datos como un todo. 26 Periodo en el cual más bien se manifiesta un movimiento ascendente de la tasa de variación interanual contraviniendo la tendencia decreciente del largo plazo. Este comportamiento podría explicarse considerando la crisis de los años 90 que supone una fuertísima caída, en términos netos de 4,36 puntos entre 1988 y 1991. 27 El tratamiento de la década de los 90, en términos de formación neta de capital fijo (FNKF), requiere, sin duda, de una mayor profundización que desentrañe las razones de este comportamiento contratendencial. Por lo tanto, a nuestro criterio, se sugiere integrar un nuevo análisis reevaluativo que, por efectos del alcance de esta investigación, queda planteado para posibles continuaciones de nuestro trabajo. Obviamente esta objeción no queda fuera de nuestro análisis.

28 Permítasenos insistir en la diferenciación de nomenclatura de la tasa de ganancia g’ (Marx) y su proxy tasa de beneficio $\Pi$.

29 Es necesario esclarecer el hecho de que la productividad laboral se encuentra fuertemente influenciada por la masa de capital (inversión) disponible para materializar el trabajo; es decir, la productividad de los trabajadores mantiene una relación cercana (pero no proporcional como se evidenciará más adelante) con el grado de mecanización de la economía.

30 Haciendo referencia a ajustes basados en el cuestionamiento del status quo salarial previo, impuesto a través de privatizaciones, desreglamentación, apertura exterior, etc., aupadas por los gobiernos de Thatcher en Reino Unido (desde 1979) y Reagan en EE. UU. (desde 1980).

31 Consideramos que dentro del periodo 1960-1980, en los tramos donde las tasas de beneficio caen, las posibles razones para estos descensos gravitan en torno a la misma explicación propuesta por Marx (a saber: mecanización de la economía, deficiente incremento en la productividad laboral, etc.). Sin embargo, lo realmente interesante de la propuesta de Marx es la ascensión de la tasa de ganancia y sus posibilidades para intentar explicar este comportamiento incremental. En otras palabras, el punto de partida es la década de los 80 porque a partir de allí la tasa crece.

32 Consecuentemente, la proporción restante pertenece a las rentas del capital (EBE/PIB).

33 Cabe señalar que la lucha de clases no es un factor exógeno, está limitado por el proceso de acumulación; es decir, hay que integrarla desde las posibilidades de apropiación de las diferentes clases sociales. 34 Aludiendo al hecho de que no hemos realizado mayores intervenciones en el procesamiento de la información contenida en el Sistema de Cuentas Nacionales. 
35 Lo cual no invalida las tesis marxistas. Recordemos que Ley del Descenso Tendencial de la Tasa de Ganancia, no implica una caída ni un ascenso real, sino más bien un conjunto de condiciones explicadas a través de sus elementos subyacentes.

36 Sobre todo en atención a los problemas de incompatibilidad de las estadísticas convencionales con las categorías marxistas.

\section{REFERENCIAS}

Arrizabalo, X. (2014). Capitalismo y economía mundial: bases teóricas y análisis empírico para la comprensión de los problemas económicos del siglo Xxi. Madrid, España: Instituto Marxista de Economía.

Astarita, R. (agosto, 2009). La tasa de ganancia y las crisis en el análisis marxista (Borrador). Rolando Astarita. Recuperado de http://rolandoastarita. com/novDebate\% 2oteoria\% 2omarxista\% 2ode\% 2ola\% 2ocrisis.htm

Cámara, S. (2003). Tendencias de la Rentabilidad y de la Acumulación de Capital en España: 1954-2001. (Tesis doctoral) Universidad Complutense de Madrid, Madrid, España.

Cámara, S., \& Mariña, A. (2010). Naturaleza y perspectivas de la actual crisis: una caracterización marxista de largo plazo. Política y cultura, (34), 7-31.

Duménil, G., y Lévy, D. (1993). The economics of the profit rate: Competition, crises, and historical tendencies in capitalism. Hampshire, England: Edward Elgar.

Gill, L. (2002). Fundamentos y límites del capitalismo. Madrid, España: Trotta.

Guerrero, D. (1989). Acumulación de Capital, Distribución de la Renta y Crisis de Rentabilidad en España (1954-1987). (Tesis doctoral) Universidad Complutense de Madrid, Madrid, España.

Guerrero, D. (1997). Un Marx imposible: el marxismo sin teoría laboral del valor. Investigación Económica, $(\mathrm{s} / \mathrm{n}), 105-143$.

Guerrero, D. (2006). La explotación: trabajo y capital en España (1954-2001). Madrid, España: El Viejo Topo.

Guerrero, D. (2008). Un resumen completo de «El capital» de Marx. Madrid, España: Maia.

Heller, P. (2011). Tasa de ganancia y crisis mundial. Hic Rhodus. Crisis capitalista, polémica y controversias, (1), 47-64.

Husson, M. (2008). La subida tendencial de la tasa de explotación. Viento Sur, (s/n), 562-563.

Katz, C. (2002). Una interpretación contemporánea de la ley de la tendencia decreciente de la tasa de ganancia. Filosofía, política y economía en el Laberinto, (8), 85-100.

Maito, E. (2013). La tasa de ganancia en Chile, 1986-2009. Razón y Revolución, (24), 43-63.

Maito, E. (2014). La transitoriedad histórica del capital. La tendencia descendente de la tasa de ganancia desde el siglo XIX. Razón y Revolución, (26), 129-159.

Mandel, E. (1985). El capital: cien años de controversias en torno a la obra de Karl Marx. México DF, México: Siglo Xxi.

Marx, K. (1859). Contribución a la Crítica de la Economía Política. Madrid: Siglo xxi.

Marx, K. (1861-1863). Teorías sobre la Plusvalía. Tomo IV de El Capital, Volumen I. México DF, México: Fondo de Cultura Económica.

Marx, K. (1867). El Capital. Crítica de la Economía Política. Libro I: El Proceso de Producción del Capital (3 tomos). Madrid, España: Akal.

Marx, K. (1885). El Capital. Crítica de la Economía Política. Libro II: El Proceso de Circulación del Capital (2 tomos). Madrid, España: Akal. 
Marx, K. (1894). El Capital. Crítica de la Economía Política. Libro III: El Proceso Global de la Producción Capitalista (3 tomos). Madrid, España: Akal.

Mateo, J. (2004). Salarios y renta nacional: teoría y evidencia empírica para la economía venezolana. Ensayos de Economía, 14(25), 32-63.

Mateo, J. (2006). Crisis de rentabilidad, acumulación de capital y distribución de la renta en la economía de México. Ponencia presentada en Encuentro de Latinoamericanistas Españoles Santander, España.

Mateo, J. (2006). Mecanización y distribución del ingreso en la crisis de rentabilidad de la economía mexicana. Economía Gestión y Desarrollo, (5), 7-40.

Mateo, J. (2007). La tasa de ganancia del capital: caracterización teórica y propuesta empírica, Oikos: Revista de la Escuela de Administración y Economía, (23), 93-118.

Mateo, J. (2007). La tasa de Ganancia en México, 1970-2003. Análisis de la Crisis de rentabilidad a partir de la Composición del Capital y la Distribución del Ingreso. (Tesis doctoral) Universidad Complutense de Madrid, Madrid, España.

Mateo, J. (2007). La tasa de Ganancia en México, 1970-2003. Análisis de la Crisis de rentabilidad a partir de la Composición del Capital y la Distribución del Ingreso. (Tesis doctoral). Universidad Complutense de Madrid, Madrid.

Moseley, F. (1991). The falling rate of profit in the postwar United States economy. Macmillan Academic and Professional. Recuperado de https://www.mtholyoke.edu/ fmoseley/working\%2opapers/ PWCRISIs.pdf

Robles, M. (2005, julio). El Concepto de Capital y la Determinación de las Tasas General y Uniforme de Ganancia: Una Reinterpretación. Ponencia presentada en la II Conferencia Marx: sobre el Concepto de Capital. México DF, México.

Rubin, I. (1923). Ensayo sobre la Teoría Marxista del Valor, Cuadernos de Pasado y Presente. Buenos Aires, Argentina: Siglo xxI.

Shaikh, A. (1984). Cuentas de ingreso nacional y categorías marxistas. Economía, teoría y práctica, (4), 3-58.

Shaikh, A. (1990). Valor, acumulación y crisis: Ensayos de Economía Política. Bogotá, Colombia: Tercer Mundo.

Shaikh, A. (2011). Tasa Decreciente de Ganancia y Crisis Económica de los Estados Unidos. Realidad Económica, (138), 119-148.

Shaikh, A., y Tonak, E. (1994). Measuring the wealth of nations: the political economy of national accounts. New York, UsA: Cambridge Books. 